Article

\title{
Heterocyclic-2-carboxylic Acid (3-Cyano-1,4-di- $N$ - oxidequinoxalin-2-yl)amide Derivatives as Hits for the Development of Neglected Disease Drugs
}

Saioa Ancizu ${ }^{1}$, Elsa Moreno ${ }^{1}$, Enrique Torres ${ }^{1}$, Asunción Burguete ${ }^{1}$, Silvia Pérez-Silanes ${ }^{1}$, Diego Benítez ${ }^{2}$, Raquel Villar ${ }^{1}$, Beatriz Solano ${ }^{1}$, Adoración Marín ${ }^{1}$, Ignacio Aldana ${ }^{1}$, Hugo Cerecetto $^{2}$, Mercedes González ${ }^{2, *}$ and Antonio Monge ${ }^{1, *}$

1 Unidad en Investigación y Desarrollo de Medicamentos, Centro de Investigación en

Farmacobiología Aplicada (CIFA), University of Navarra, C/Irunlarrea s/n, 31008 Pamplona, Spain; E-mails: sancizupere@alumni.unav.es (S.A.), emoreno4@alumni.unav.es (E.M)

2 Laboratorio de Química Orgánica, Facultad de Ciencias/Facultad de Química, Universidad de la República, Iguá 4225, 11400 Montevideo, Uruguay; E-mails: dbenitez@fq.edu.uy (D.B.), hcerecet@fq.edu.uy (H.C.)

* Authors to whom correspondence should be addressed; E-mails: cifa@unav.es (A.M.), megonzal@fq.edu.uy (M.G.)

Received: 2 June 2009; in revised form: 15 June 2009 / Accepted: 17 June 2009 /

Published: 22 June 2009

\begin{abstract}
Neglected diseases represent a major health problem. It is estimated that one third of the world population is infected with tuberculosis (TB). Besides TB, Chagas disease, affects approximately 20 million people. Quinoxalines display great activities against TB and Chagas. Forty new quinoxaline 1,4-di- $N$-oxide derivatives have been prepared and tested against $M$. tuberculosis and T. cruzi. Carboxylic acid quinoxaline 1,4-di- $N$-oxides (CAQDOs) 5 and 17 showed MIC values on the same order as the reference antituberculosis drug, rifampicin. Meanwhile, CAQDOs 12 and 22 presented $\mathrm{IC}_{50}$ values in the same order as the anti-chagasic drug, nifurtimox.
\end{abstract}

Keywords: quinoxaline; neglected diseases; Mycobacterium tuberculosis; Trypanosoma cruzi 


\section{Introduction}

Mycobacterium tuberculosis (M. tuberculosis), and to a lesser extent M. bovis and M. africanum, can cause a chronic and fatal condition in humans known as tuberculosis (TB). Until about 50 years ago, this disease was considered virtually incurable. The discovery of several active anti-TB agents heralded a new age of anti-TB chemotherapy. Therefore, TB was considered to be a curable disease. Unfortunately, in only a few years, it became apparent that the use of these drugs as single agents led to rapid drug resistance and treatment failures among a substantial number of patients. It was quickly realized, however, that the development of resistance could be forestalled or prevented through treatment with several active agents in a combination regimen. Of particular concern is the development of multi-drug-resistant forms of the disease (MDR-TB), defined as forms resistant to two or more of the front line anti-TB agents. These forms of the disease are most often fatal and are difficult and expensive to treat. It is estimated that one third of the world's population is infected with $\mathrm{TB}$, with about eight million new cases annually. Of these cases, 3.1 million die annually, more deaths than those caused by any other single infectious disease. TB is the leading killer of youths, women, and AIDS patients in the world [1,2]. HIV-infected patients have an elevated risk of tuberculosis, and such active infectious process may enhance HIV replication and increase the risk of death. It has been estimated that up to 50 million people are infected with drug-resistant forms of TB. Due to the fact that the current frontline therapy for TB consists of administering three different drugs (the antibiotic rifampicin, RIF, and the azaheterocycles isoniazid and pyrazinamide, Isnz and Pyzd, Scheme 1) over an extended period of time as well as the problems that arise due to MDR-TB, it is necessary to develop new, potent, fast-acting anti-tuberculosis drugs with low-toxicity profiles for treating drug resistant forms of the disease that can be given in conjunction with drugs used to treat HIV infections $[3,4]$.

Besides TB, the parasitic diseases represent a major health problem in Third World countries. More specifically, Chagas disease, or American trypanosomiasis, caused by the protozoan Trypanosoma cruzi (T. cruzi), is the largest parasitic disease burden in the American continents. It affects approximately 20 million people from the southern United States to southern Chile. Even though the enforcement of public health programs towards vector elimination in some Latin American countries has decreased the incidence of new infections, the disease is still endemic in large areas. Every year, 21,000 people die from this parasitosis and over 200, 000 new cases arise [5]. Currently, there are only two clinically used drugs, nifurtimox (Nfx, Scheme 1) and benznidazole. Both are nitroheterocyclic compounds that possess important toxic effects and relative clinical efficacy; therefore, the pharmacotherapy of Chagas disease is very deficient and there is an urgent need for the development of safe and effective drugs [6].

Quinoxalines, including their fused-ring derivatives, display diverse pharmacological activities and more specifically, their 1,4-di- $N$-oxides have demonstrated excellent activities as antiviral, anticancer, antibacterial, and antiparasitic agents [7]. In this sense, our group published several studies in which the synthesis and biological evaluation of a large amount of quinoxaline 1,4-di- $N$-oxides (QDO) have been described. We have focused our recent efforts on the development of QDO with activities against the agents responsible of some of the well-known neglected diseases. We have found QDO with good in vitro selectivity against M. tuberculosis (i.e. parent compounds 1 and 2, Scheme 1) [8-16] and 
against T. cruzi (i.e. parent compound 3, Scheme 1) [17,18], and in both cases, recognizing some structural requirements for optimal activity [12-20].

Galactofuranose is an essential component of the mycobacterial cell wall, not found in man; UDPgalactofuranose is biosynthesized from UDP-galactopyranose using the enzyme UDP-galactose mutase (Glf). In 2004, Tangallapally et al. discovered that nitrofuryl derivatives have the requirements for optimum inhibition of Glf activity (i.e. compound 4, Scheme 1) [4]. In addition, the nitrofuryl moiety is present in a large number of anti-T. cruzi agents acting via a nitroreduction process, generating redox cycling at different levels [21]. Based on these structural features, we have designed a new series of quinoxaline 1,4-di- $N$-oxide derivatives containing a nitrofuryl side chain as potential anti-neglected diseases agents. More specifically, we have designed new hybrid QDO with potential anti-tubercular activity combining some previous structural features, amide and cyano QDO substituents, and the heteroaryl retro-amide moieties. In order to determine the action of the 5nitrofuryl moiety, we synthesized another analogue series by substituting this group by a 5-nitrothienyl one, and in order to determine the influence of the nitro group, another two series were designed, furyl and thienyl side chains (Table 1). Moreover, the same designed compounds could also act as hybrid potential anti-T. cruzi agents because they are QDO that have maintained the 3-cyano and 2-NH, as retro-amide moieties, with the extra-heteroaryl substituents (5-nitrofuryl, 5-nitrothienyl, furyl, and thienyl groups).

Scheme 1. Design of new QDOs as potential drugs against neglected diseases.

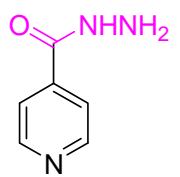

Isnz<smiles>NC(=O)c1cnccn1</smiles>

Pyzd<smiles>CC1CS(=O)(=O)CCN1/N=C/c1ccc([N+](=O)[O-])o1</smiles>

Nfx

A name could not be generated for this structure.<smiles>Cc1ccccc1NC(=O)c1c(C)[n+]([O-])c2ccc(Cl)cc2[n+]1[O-]</smiles>

1<smiles>Cc1ccc2c(c1)[n+]([O-])c(-c1ccc(F)cc1)c(C#N)[n+]2[O-]</smiles>

2<smiles>N#Cc1c(Nc2ccccc2)[n+](O)c2ccc(Cl)cc2[n+]1O</smiles>

3<smiles>COc1ccc(NC(=O)c2ccc([N+](=O)[O-])o2)cc1Cl</smiles>

4

Design

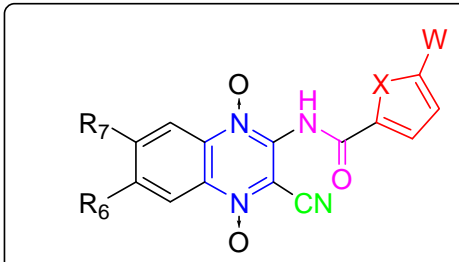




\section{Results and Discussion}

We have prepared forty new heterocyclic-2-carboxylic acid (3-cyano-1,4-di- $N$-oxidequinoxalin-2yl)-amide derivatives (CAQDOs). The benzofuroxane starting compounds, (BFX, I, Scheme 2), have been prepared using previously described methods [11,22]. The 3-amine-1,4-di- $N$-oxide quinoxaline2-carbonitrile derivatives (cyanoamines, II) were obtained by the Beirut reaction from the corresponding BFX, with malononitrile using $N, N$-dimethylformamide (DMF) as solvent and triethylamine as catalyst [23]. Finally, the new CAQDOs were synthesized using two different routes, I and II (Scheme 2) [4, 24]. Furyl and thienyl derivatives 5-24 were obtained by reaction between intermediates II with an excess of the corresponding, commercially available, heteroaryl-2-carbonyl chloride. Another synthetic route was optimized for the synthesis of 5-nitrofuryl and 5-nitrothienyl derivatives 25-44. In these cases, the reaction was carried out by condensation between intermediates II and the corresponding carboxylic acid, 5-nitrofuryl and 5-nitrothienyl carboxylic acid, activated in the presence of EDCI and DMAP and DMF as solvent.

Scheme 2. Synthetic route to heterocyclic-2-carboxylic acid (3-cyano-1,4-di- $N$ oxidequinoxalin-2-yl)-amide derivatives 5-44.<smiles>[R6]c1cc2no[n+]([O-])c2cc1[R6]</smiles>

I
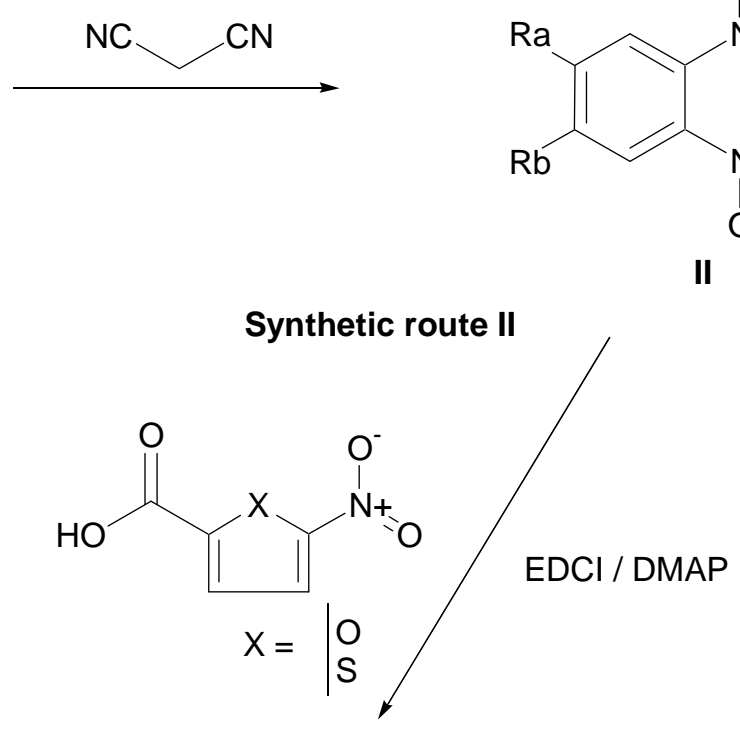

II
$\mathrm{Ra}, \mathrm{Rb}=\mathrm{H}$ $\mathrm{CH}_{3}$ $\mathrm{Cl}$ $\mathrm{CH}_{3} \mathrm{O}$ $\mathrm{F}$ $\mathrm{CF}_{3}$

Synthetic route I

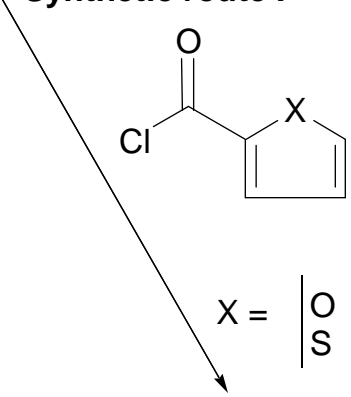<smiles>[R10]c1cc2c(cc1[R6])[n+]([O-])c(NC(=O)c1[X]c([N+](=O)[O-])cc1)c(C#N)[n+]2[O-]</smiles>

(25-44)<smiles>[R6]c1cc2c(cc1[R])[n+]([O-])c(C#N)c(N)[n+]2[O-]</smiles>

(5-24) 
In order to carry out an in depth QSAR study, CAQDO were un-substituted or substituted in positions 6 and 7 by chloro- or fluoro- or trifluoromethyl-moiety as electron-withdrawing groups and by methyl- or methoxy-moiety as electron-releasing groups. When the new CAQDOs were prepared from monosubstituted-BFX, a mixture of positional isomers was obtained. Generally, it could be observed that the 6-substituted isomer prevailed over the 7-substituted isomer [25]. When the substituent was a methoxy moiety, a regio-specific reaction was achieved because only 6-isomer was isolated. In the other case, when 5-trifluoromethyl-BFX was reacted, the two CAQDO-isomers were obtained in similar proportions and separated by chromatography.

The new developed CAQDOs were subjected to the following set of tests: i) determination of the MICs, in $\mu \mathrm{g} / \mathrm{mL}$, against $M$. tuberculosis H37Rv strain and, ii) determination of the percentage of growth inhibition, at $25 \mu \mathrm{M}$, and $\mathrm{IC}_{50}$ values, in $\mu \mathrm{M}$, against $T$. cruzi Tulahuen 2 strain (Table 1).

With regard to the anti-M. tuberculosis evaluations, the CAQDO 5 and $\mathbf{1 7}$ were identified as the most active derivatives against H37Rv strain, with MIC values on the same order as the reference compound, RIF (Table 1). Some structure-activity relationships could be established; in general, thienyl-derivatives are more active than furyl derivatives (cf. the anti-M. tuberculosis activities of 16 and 6, 19 and 9, 23 and 13, 24 and 14, 38 and 28, 43 and 33, or 44 and 34), whereas the effect of the 5nitro substitution is clear only for the thienyl series (cf. the anti-M. tuberculosis activities of $\mathbf{3 5}$ and 15, 38 and 18, 43 and 23, or 44 and 24), with the 5-nitrothienyl-derivatives being more active than the unsubstituted ones. For this biological activity we were unable to find relationships between this and the electronic characteristics of benzo-substituent on the quinoxaline heterocycle. However, it could be pointed out, bearing in mind derivatives 29-32, that the mono-halogen substitution produce compounds more actives than the di-halogen substituted ones furthermore chlorine-substitution is better for the activity than fluorine-substitution. Considering the couple of derivatives 13 and 14, 23 and 24, 33 and 34, and 43 and 44 the 7-trifluoromethyl-substitution produces more active compounds.

With regard to the anti-T. cruzi evaluations, the difluoro substituted CAQDOs 12 and 22 were identified as the most active derivatives against the Tulahuen 2 strain, with $\mathrm{IC}_{50}$ values on the same order as the reference compound, Nfx. Moreover, compound 22 was found more active than the parent compound 3 (Scheme 1, Table 1). Similar to M. tuberculosis, the thienyl-derivatives are more active than furyl derivatives (compare anti-T. cruzi activities of 22 and 12), unlike in the case of $M$. tuberculosis, in which the influence of the 5-nitro substitution is clear, with the 5-nitro-substituted derivatives being less active than the un-substituted derivatives (compare anti-T. cruzi activities of 42 and 22). In T. cruzi findings, some relationships between the electronic characteristics of benzosubstituent, on the quinoxaline heterocycle, and the activity could be established; for example, when the electron-withdrawing property increases, the activity increases (compare activity of compound $\mathbf{1 0}$ with 8 or 7, 12 and 10, 22 and 21, or 40 and 39). The hybridization process, pharmacophore quinoxaline dioxide plus pharmacophore nitrofurane, does not produce active compounds. 
Table 1. Biological characterization of the forty new quinoxaline 1,4-di- $N$-oxides.

\begin{tabular}{|c|c|c|c|c|c|c|}
\hline Cpd. & $\mathbf{X}$ & $\bar{W}$ & R7 & R6 & $\operatorname{MIC}^{\mathrm{a}}(\mu \mathrm{g} / \mathrm{mL})$ & $\% \mathrm{GI}^{\mathrm{b}}$ \\
\hline 5 & $\mathrm{O}$ & $\overline{\mathrm{H}}$ & $\mathrm{H}$ & $\overline{\mathrm{H}}$ & 0.977 & 3.1 \\
\hline 6 & $\mathrm{O}$ & $\mathrm{H}$ & $\mathrm{H}$ & $\mathrm{CH}_{3}$ & 8.843 & 11.6 \\
\hline 7 & $\mathrm{O}$ & $\mathrm{H}$ & $\mathrm{CH}_{3}$ & $\mathrm{CH}_{3}$ & $\mathrm{NT}^{\mathrm{c}}$ & 13.1 \\
\hline 8 & $\mathrm{O}$ & $\mathrm{H}$ & $\mathrm{H}$ & $\mathrm{OCH}_{3}$ & NT & 9.5 \\
\hline 9 & $\mathrm{O}$ & $\mathrm{H}$ & $\mathrm{H}$ & $\mathrm{Cl}$ & 15.361 & NT \\
\hline 10 & $\mathrm{O}$ & $\mathrm{H}$ & $\mathrm{Cl}$ & $\mathrm{Cl}$ & NT & 31.6 \\
\hline 11 & $\mathrm{O}$ & $\mathrm{H}$ & $\mathrm{H}$ & $\mathrm{F}$ & NT & 19.8 \\
\hline 12 & $\mathrm{O}$ & $\mathrm{H}$ & $\mathrm{F}$ & $\mathrm{F}$ & 4.700 & $\begin{array}{c}53.1 \\
\left(\mathrm{IC}_{50}=19.2\right)\end{array}$ \\
\hline 13 & $\mathrm{O}$ & $\mathrm{H}$ & $\mathrm{CF}_{3}$ & $\mathrm{H}$ & 17.903 & NT \\
\hline 14 & $\mathrm{O}$ & $\mathrm{H}$ & $\mathrm{H}$ & $\mathrm{CF}_{3}$ & 24.220 & NT \\
\hline 15 & $\mathrm{~S}$ & $\mathrm{H}$ & $\mathrm{H}$ & $\mathrm{H}$ & 5.381 & 19.0 \\
\hline 16 & $\mathrm{~S}$ & $\mathrm{H}$ & $\mathrm{H}$ & $\mathrm{CH}_{3}$ & 2.082 & 16.4 \\
\hline 17 & $\mathrm{~S}$ & $\mathrm{H}$ & $\mathrm{CH}_{3}$ & $\mathrm{CH}_{3}$ & 1.190 & 13.5 \\
\hline 18 & $\mathrm{~S}$ & $\mathrm{H}$ & $\mathrm{H}$ & $\mathrm{OCH}_{3}$ & 48.909 & 15.1 \\
\hline 19 & $\mathrm{~S}$ & $\mathrm{H}$ & $\mathrm{H}$ & $\mathrm{Cl}$ & 2.470 & NT \\
\hline 20 & $\mathrm{~S}$ & $\mathrm{H}$ & $\mathrm{Cl}$ & $\mathrm{Cl}$ & 13.612 & NT \\
\hline 21 & $\mathrm{~S}$ & $\mathrm{H}$ & $\mathrm{H}$ & $\mathrm{F}$ & 1.474 & 27.5 \\
\hline 22 & $\mathrm{~S}$ & $\mathrm{H}$ & $\mathrm{F}$ & $\mathrm{F}$ & NT & $\begin{array}{c}92.4 \\
\left(\mathrm{IC}_{50}=10.8\right)\end{array}$ \\
\hline 23 & $\mathrm{~S}$ & $\mathrm{H}$ & $\mathrm{CF}_{3}$ & $\mathrm{H}$ & 4.576 & NT \\
\hline 24 & $\mathrm{~S}$ & $\mathrm{H}$ & $\mathrm{H}$ & $\mathrm{CF}_{3}$ & 9.177 & NT \\
\hline 25 & $\mathrm{O}$ & $\mathrm{NO}_{2}$ & $\mathrm{H}$ & $\mathrm{H}$ & NT & 0.7 \\
\hline 26 & $\mathrm{O}$ & $\mathrm{NO}_{2}$ & $\mathrm{H}$ & $\mathrm{CH}_{3}$ & NT & 0.0 \\
\hline 27 & $\mathrm{O}$ & $\mathrm{NO}_{2}$ & $\mathrm{CH}_{3}$ & $\mathrm{CH}_{3}$ & NT & 0.0 \\
\hline 28 & $\mathrm{O}$ & $\mathrm{NO}_{2}$ & $\mathrm{H}$ & $\mathrm{OCH}_{3}$ & 14.493 & NT \\
\hline 29 & $\mathrm{O}$ & $\mathrm{NO}_{2}$ & $\mathrm{H}$ & $\mathrm{Cl}$ & 5.811 & NT \\
\hline 30 & $\mathrm{O}$ & $\mathrm{NO}_{2}$ & $\mathrm{Cl}$ & $\mathrm{Cl}$ & 13.573 & NT \\
\hline 31 & $\mathrm{O}$ & $\mathrm{NO}_{2}$ & $\mathrm{H}$ & $\mathrm{F}$ & 21.675 & NT \\
\hline 32 & $\mathrm{O}$ & $\mathrm{NO}_{2}$ & F & $\mathrm{F}$ & 22.161 & NT \\
\hline 33 & $\mathrm{O}$ & $\mathrm{NO}_{2}$ & $\mathrm{CF}_{3}$ & $\mathrm{H}$ & 12.816 & NT \\
\hline 34 & $\mathrm{O}$ & $\mathrm{NO}_{2}$ & $\mathrm{H}$ & $\mathrm{CF}_{3}$ & 14.408 & NT \\
\hline 35 & $\mathrm{~S}$ & $\mathrm{NO}_{2}$ & $\mathrm{H}$ & $\mathrm{H}$ & 2.875 & 0.0 \\
\hline 36 & $\mathrm{~S}$ & $\mathrm{NO}_{2}$ & $\mathrm{H}$ & $\mathrm{CH}_{3}$ & NT & 11.4 \\
\hline 37 & $\mathrm{~S}$ & $\mathrm{NO}_{2}$ & $\mathrm{CH}_{3}$ & $\mathrm{CH}_{3}$ & NT & 36.4 \\
\hline 38 & $\mathrm{~S}$ & $\mathrm{NO}_{2}$ & $\mathrm{H}$ & $\mathrm{OCH}_{3}$ & 9.538 & NT \\
\hline 39 & $\mathrm{~S}$ & $\mathrm{NO}_{2}$ & $\mathrm{H}$ & $\mathrm{Cl}$ & NT & 15.0 \\
\hline 40 & $\mathrm{~S}$ & $\mathrm{NO}_{2}$ & $\mathrm{Cl}$ & $\mathrm{Cl}$ & NT & 29.8 \\
\hline 41 & $\mathrm{~S}$ & $\mathrm{NO}_{2}$ & $\mathrm{H}$ & $\mathrm{F}$ & NT & 2.5 \\
\hline 42 & $\mathrm{~S}$ & $\mathrm{NO}_{2}$ & F & $\mathrm{F}$ & NT & 9.2 \\
\hline 43 & $\mathrm{~S}$ & $\mathrm{NO}_{2}$ & $\mathrm{CF}_{3}$ & $\mathrm{H}$ & 1.695 & NT \\
\hline 44 & $\mathrm{~S}$ & $\mathrm{NO}_{2}$ & $\mathrm{H}$ & $\mathrm{CF}_{3}$ & 6.001 & NT \\
\hline 3 & - & - & - & - & - & $63.0^{\mathrm{d}}$ \\
\hline RIF & - & - & - & - & 0.125 & - \\
\hline Nfx & - & - & - & - & - & $100.0(7.7)$ \\
\hline
\end{tabular}

${ }^{a}$ Minimum inhibitory concentration against $M$. tuberculosis H37Rv. ${ }^{b}$ Percentage of growth inhibition at $25 \mu \mathrm{M}$ doses in T. cruzi Tulahuen 2 strain. ${ }^{\mathrm{c}}$ NT: Not tested. ${ }^{\mathrm{d}}$ From reference [17]. 


\section{Experimental}

\section{General}

All of the synthesized compounds were chemically characterized by thin layer chromatography (TLC), infrared (IR), proton nuclear magnetic resonance ( $\left.{ }^{1} \mathrm{H}-\mathrm{NMR}\right)$, mass spectra (MS) and elemental microanalyses (CHN). Alugram SIL G/UV254 (Layer: $0.2 \mathrm{~mm}$ ) (Macherey-Nagel GmbH \& Co. KG., Düren, Germany) was used for TLC and Silica gel 60 (0.040-0.063 mm, Merck) was used for Flash Column Chromatography. The ${ }^{1} \mathrm{H}-\mathrm{NMR}$ spectra were recorded on a Bruker 400 Ultrashield instrument (400 MHz), using TMS as internal standard and with DMSO- $\mathrm{d}_{6}$ as solvent; the chemical shifts are reported in ppm $(\delta)$ and coupling constants $(J)$ values are given in Hertz $(\mathrm{Hz})$. Signal multiplicities are represented by: s (singlet), bs (broad singlet), d (doublet), t (triplet), q (quadruplet), dd (double doublet) and $\mathrm{m}$ (multiplet). The IR spectra were recorded on a Nicolet Nexus FTIR (Thermo, Madison, USA) in $\mathrm{KBr}$ pellets. Elemental microanalyses were obtained on a CHN-900 Elemental Analyzer (Leco, Tres Cantos, Spain) from vacuum-dried samples. The analytical results for $\mathrm{C}, \mathrm{H}$ and $\mathrm{N}$, were within \pm 0.5 of the theoretical values. Chemicals were purchased from Panreac Química S.A. (Barcelona, Spain), Sigma-Aldrich Química, S.A. (Alcobendas, Spain), Acros Organics (Janssen Pharmaceutical, Geel, Belgium) and Lancaster (Bischheim-Strasbourg, France).

\section{General procedure for the synthesis of cyanoamines II}

Malononitrile (18.0 mmol) was added to a solution of the appropriate benzofuroxane (I, $15.0 \mathrm{mmol})$ in DMF $(10 \mathrm{~mL})$. The mixture was allowed to stand at $0{ }^{\circ} \mathrm{C}$. Triethylamine was added dropwise $(1.5$ $\mathrm{mL}$ ), and the reaction mixture was stirred at room temperature in darkness for 1-3 days. The precipitate was filtered off and washed by adding diethyl ether affording the target compound. The obtained red solid was used in the next step without further purification.

General procedure for the synthesis of furan-2-carboxylic acid (3-cyano-1,4-di-N-oxide-quinoxalin-2yl)-amide derivatives and thiophene-2-carboxylic acid (3-cyano-1,4- $\mathrm{N}$-oxide-quinoxalin-2-yl)-amide derivatives 5-24

The corresponding 3-amino-1,4-di- $N$-oxide-quinoxaline-2-carbonitrile II $(2 \mathrm{mmol})$ is dissolved in acetonitrile $(100 \mathrm{~mL})$ and triethylamine $(0.4 \mathrm{~mL})$ was added at room temperature under stirring and anhydrous conditions. After cooling the reaction mixture with an ice bath, 2-furoyl chloride or 2tiophenecarbonyl chloride $(2.2 \mathrm{mmol})$ are added. The reaction mixture is stirred for $9 \mathrm{~h}$ at room temperature. The obtained solid is filtered and EtOAc $(400 \mathrm{~mL})$ added to the filtrate. The organic phase is extracted, first with $\mathrm{HCl} 10 \%$ and then with water. The organic phase is dried with anhydrous $\mathrm{Na}_{2} \mathrm{SO}_{4}$ and filtered. The solvent is removed in vacuo and the resulting residue is precipitated with diethyl ether, and then filtered in order to obtain a yellow-orange solid.

Furan-2-carboxylic acid (3-cyano-1,4-di-N-oxidequinoxalin-2-yl)amide (5). Yield $64.4 \% ;{ }^{1} \mathrm{H}-\mathrm{NMR} \delta$ ppm: 11.43 (bs, $1 \mathrm{H}, \mathrm{NH})$; 8.53-8.48 (m, 2H, $\left.\mathrm{H}_{8}+\mathrm{H}_{5}\right)$; 8.13-8.01 (m, 3H, $\mathrm{H}_{6}+\mathrm{H}_{7}+\mathrm{H}_{5}$ ); 7.60 (d, 1H, $\mathrm{H}_{3^{\prime}}$, $\left.J_{3^{\prime}-4^{\prime}}=3.5 \mathrm{~Hz}\right) ; 6.81\left(\mathrm{dd}, 1 \mathrm{H}, \mathrm{H}_{4^{\prime}}, J_{4^{\prime}-5}{ }^{\prime}=1.7 \mathrm{~Hz}\right) ; \mathrm{IR} v \mathrm{~cm}^{-1}: 3,237(\mathrm{~m}, \mathrm{NH}) ; 2,236(\mathrm{w}, \mathrm{C} \equiv \mathrm{N}) ; 1,686(\mathrm{~s}$, 
$\mathrm{C}=\mathrm{O}) ; 1,335\left(\mathrm{~s}, \mathrm{~N}^{+} \mathrm{O}^{-}\right)$; Anal. Calc. for $\mathrm{C}_{14} \mathrm{H}_{8} \mathrm{~N}_{4} \mathrm{O}_{4}: \mathrm{C}: 56.76 \%$; $\mathrm{H}: 2.72 \%$; $\mathrm{N}: 18.91 \%$. Found: $\mathrm{C}$ : 56.73\%; H: 3.13\%; N: 18.93\%.

Furan-2-carboxylic acid (3-cyano-1,4-di- $N$-oxide-6-methylquinoxalin-2-yl)amide (6). Yield $63.0 \%$; ${ }^{1} \mathrm{H}-\mathrm{NMR} \delta$ ppm: 11.37 (bs, $\left.1 \mathrm{H}, \mathrm{NH}\right) ; 8.42\left(\mathrm{~d}, 1 \mathrm{H}, \mathrm{H}_{8}, J_{8-7}=8.8 \mathrm{~Hz}\right) ; 8.31\left(\mathrm{~d}, 1 \mathrm{H}, \mathrm{H}_{5}, J_{5-7}=1.3 \mathrm{~Hz}\right.$ ); $8.10\left(\mathrm{~d}, 1 \mathrm{H}, \mathrm{H}_{5^{\prime}}, J_{5^{\prime}-4^{\prime}}=1.7 \mathrm{~Hz}\right) ; 7.95\left(\mathrm{dd}, 1 \mathrm{H}, \mathrm{H}_{7}\right) ; 7.69\left(\mathrm{~d}, 1 \mathrm{H}, \mathrm{H}_{3^{\prime}}, J_{3^{\prime}-4^{\prime}}=3.6 \mathrm{~Hz}\right), 6.81\left(\mathrm{dd}, 1 \mathrm{H}, \mathrm{H}_{4^{\prime}}\right)$, $2.61\left(\mathrm{~s}, 3 \mathrm{H}, \mathrm{CH}_{3}\right)$; IR $v \mathrm{~cm}^{-1}: 3,114(\mathrm{~m}, \mathrm{NH}) ; 2,238(\mathrm{w}, \mathrm{C} \equiv \mathrm{N}) ; 1,692(\mathrm{~s}, \mathrm{C}=\mathrm{O}) ; 1,325\left(\mathrm{~s}, \mathrm{~N}^{+} \mathrm{O}^{-}\right)$; Anal. Calc. for $\mathrm{C}_{15} \mathrm{H}_{10} \mathrm{~N}_{4} \mathrm{O}_{4}$ : C: $58.07 \%$; H: 3.25\%; N: 18.06\%. Found: C: $57.95 \%$; H: $3.27 \%$; N: 18.43\%.

Furan-2-carboxylic acid (3-cyano-1,4-di-N-oxide-6,7-dimethylquinoxalin-2-yl)amide (7). Yield 75.3 \%; ${ }^{1} \mathrm{H}-\mathrm{NMR} \delta$ ppm: 11.33 (bs, 1H, NH); 8.31 (s, $\left.1 \mathrm{H}, \mathrm{H}_{8}\right) ; 8.28$ (s, 1H, $\left.\mathrm{H}_{5}\right) ; 8.09$ (d, 1H, $\mathrm{H}_{5^{\prime}}, J_{5^{\prime}-4^{\prime}}=1.5$ $\mathrm{Hz}) ; 7.68\left(\mathrm{~d}, 1 \mathrm{H}, \mathrm{H}_{3^{\prime}}, J_{3^{\prime}-4^{\prime}}=3.5 \mathrm{~Hz}\right) ; 6.80\left(\mathrm{dd}, 1 \mathrm{H} ; \mathrm{H}_{4^{\prime}}\right) ; 2.54-2.52\left(\mathrm{~m}, 6 \mathrm{H}, 2 \mathrm{xCH}_{3}\right) ; \mathrm{IR} v \mathrm{~cm}^{-1}: 3,285$ $(\mathrm{w}, \mathrm{NH}) ; 2,236(\mathrm{w}, \mathrm{C} \equiv \mathrm{N}) ; 1,709(\mathrm{~s}, \mathrm{C}=\mathrm{O}) ; 1,324\left(\mathrm{~s}, \mathrm{~N}^{+} \mathrm{O}^{-}\right)$; Anal. Calc. for $\mathrm{C}_{16} \mathrm{H}_{12} \mathrm{~N}_{4} \mathrm{O}_{4}: \mathrm{C}: 59.26 \% ; \mathrm{H}$ : $3.73 \%$; N: $17.28 \%$. Found: C: $59.33 \%$; H: $3.77 \%$; N: $17.30 \%$.

Furan-2-carboxylic acid (3-cyano-1,4-di-N-oxide-6-methoxyquinoxalin-2-yl)amide (8). Yield 50.5 \%; ${ }^{1} \mathrm{H}-\mathrm{NMR} \delta$ ppm: 11.31 (bs, 1H, NH); 8.44 (d, 1H, H8, J8-7 $\left.9.4 \mathrm{~Hz}\right) ; 8.09$ (s, 1H, H5); 7.78 (d, 1H, H${ }_{5}$, $\left.J_{5^{\prime}-4^{\prime}}=2.4 \mathrm{~Hz}\right) ; 7.73\left(\mathrm{~d}, 1 \mathrm{H}, \mathrm{H}_{7}\right) ; 7.67\left(\mathrm{~d}, 1 \mathrm{H}, \mathrm{H}_{3^{\prime}}, J_{3^{\prime}-4^{\prime}}=3.10 \mathrm{~Hz}\right), 6.80\left(\mathrm{dd}, 1 \mathrm{H}, \mathrm{H}_{4}\right)$ ); $4.03(\mathrm{~s}, 3 \mathrm{H}$, $\left.\mathrm{OCH}_{3}\right)$; IR $v \mathrm{~cm}^{-1}: 3,298(\mathrm{~m}, \mathrm{NH}) ; 2,241(\mathrm{w}, \mathrm{C} \equiv \mathrm{N}) ; 1,698(\mathrm{~s}, \mathrm{C}=\mathrm{O}) ; 1,321\left(\mathrm{~s}, \mathrm{~N}^{+} \mathrm{O}^{-}\right)$; Anal. Calc. for $\mathrm{C}_{15} \mathrm{H}_{10} \mathrm{~N}_{4} \mathrm{O}_{5}$ : C: $55.22 \%$; H: 3.09\%; N: 17.17\%. Found: C: $54.87 \%$; H: 3.09\%; N: $16.79 \%$.

Furan-2-carboxylic acid (3-cyano-6-chloro-1,4-di- $N$-oxidequinoxalin-2-yl)amide (9). Yield $6.1 \% ;{ }^{1} \mathrm{H}$ NMR $\delta$ ppm: 11.47 (bs, 1H, NH); 8.51-8.50 (m, 2H, $\left.\mathrm{H}_{8}+\mathrm{H}_{5}\right) ; 8.14$ (dd, $1 \mathrm{H}, \mathrm{H}_{7}, J_{7-8}=9.1 \mathrm{~Hz}, J_{7-5}=2.3$ $\mathrm{Hz}) ; 8.10\left(\mathrm{~d}, 1 \mathrm{H}, \mathrm{H}_{5^{\prime}}, J_{5^{\prime}-4}=1.7 \mathrm{~Hz}\right) ; 7.70\left(\mathrm{~d}, 1 \mathrm{H}, \mathrm{H}_{3^{\prime}}, J_{3^{\prime}-4^{\prime}}=3.6 \mathrm{~Hz}\right) ; 6.81\left(\mathrm{dd}, 1 \mathrm{H}, \mathrm{H}_{4^{\prime}}, J_{4^{\prime}-3^{\prime}}=3.6 \mathrm{~Hz}\right.$, $\left.J_{4^{\prime}-5},=1.7 \mathrm{~Hz}\right)$; IR $v \mathrm{~cm}^{-1}: 3,288(\mathrm{~m}, \mathrm{NH}) ; 2,241(\mathrm{w}, \mathrm{C} \equiv \mathrm{N}) ; 1,692(\mathrm{~s}, \mathrm{C}=\mathrm{O}) ; 1,320\left(\mathrm{~s}^{-} \mathrm{N}^{+} \mathrm{O}^{-}\right)$; Anal Calc. for $\mathrm{C}_{14} \mathrm{H}_{7} \mathrm{ClN}_{4} \mathrm{O}_{4}$ : C: $50.58 \%$; H: 2.13\%; N: 16.94\%. Found: 50.91\%; H: 2.23\%; N: $16.69 \%$.

Furan-2-carboxylic acid (3-cyano-6,7-dichloro-1,4-di-N-oxidequinoxalin-2-yl)amide (10). Yield 51.0 $\%$; ${ }^{1} \mathrm{H}-\mathrm{NMR} \delta$ ppm: 11.56 (bs, $\left.1 \mathrm{H}, \mathrm{NH}\right) ; 8.72\left(\mathrm{~s}, 2 \mathrm{H}, \mathrm{H}_{8}+\mathrm{H}_{5}\right) ; 8.11\left(\mathrm{dd}, 1 \mathrm{H}, \mathrm{H}_{5^{\prime}}, J_{5^{\prime}-4^{\prime}}=1.7 \mathrm{~Hz}, J_{5^{\prime}-3^{\prime}}=\right.$ $0.7 \mathrm{~Hz}) ; 7.72\left(\mathrm{~d}, 1 \mathrm{H}, \mathrm{H}_{3^{\prime}}, J_{3^{\prime}-4^{\prime}}=3.6 \mathrm{~Hz}\right) ; 6.81\left(\mathrm{dd}, 1 \mathrm{H}, \mathrm{H}_{4}\right) ; \mathrm{IR} v \mathrm{~cm}^{-1}: 3,275(\mathrm{~m}, \mathrm{NH}) ; 2,232$ (w, $\mathrm{C} \equiv \mathrm{N}) ; 1,702(\mathrm{~s}, \mathrm{C}=\mathrm{O}) ; 1,336\left(\mathrm{~s}, \mathrm{~N}^{+} \mathrm{O}^{-}\right)$; Anal. Calc. for $\mathrm{C}_{14} \mathrm{H}_{6} \mathrm{Cl}_{2} \mathrm{~N}_{4} \mathrm{O}_{4}: \mathrm{C}: 45.04 \% ; \mathrm{H}: 1.61 \%$; : 15.01\%. Found: C: 45.34\%; H: 1.75\%; N: 15.18\%.

Furan-2-carboxylic acid (3-cyano-1,4-di-N-oxide-6-fluoroquinoxalin-2-yl)amide (11). Yield 57.8 \%; ${ }^{1} \mathrm{H}-\mathrm{NMR} \delta$ ppm: 11.44 (bs, $\left.1 \mathrm{H}, \mathrm{NH}\right) ; 8.59$ (dd, $\left.1 \mathrm{H}, \mathrm{H}_{8}, J_{8-\mathrm{F}}=5.0 \mathrm{~Hz}\right) ; 8.30$ (dd, $1 \mathrm{H}, \mathrm{H}_{5}, J_{5-\mathrm{F}}=8.6 \mathrm{~Hz}$, $\left.J_{5-7}=2.7 \mathrm{~Hz}\right) ; 8.10\left(\mathrm{~d}, 1 \mathrm{H}, \mathrm{H}_{5^{\prime}}, J_{5^{\prime}-4^{\prime}}=1.5 \mathrm{~Hz}\right) ; 8.03\left(\mathrm{ddd}, 1 \mathrm{H}, \mathrm{H}_{7}, J_{7-\mathrm{F}}=7.9 \mathrm{~Hz}\right) ; 7.70\left(\mathrm{~d}, 1 \mathrm{H}, \mathrm{H}_{3^{\prime}}, J_{3^{\prime}-}\right.$ $\left.4^{\prime}=3.6 \mathrm{~Hz}\right) ; 6.81\left(\mathrm{dd}, 1 \mathrm{H}, \mathrm{H}_{4}\right)$; IR $v \mathrm{~cm}^{-1}: 3,263(\mathrm{~m}, \mathrm{NH}) ; 2,239(\mathrm{w}, \mathrm{C} \equiv \mathrm{N}) ; 1,681(\mathrm{~s}, \mathrm{C}=\mathrm{O}) ; 1,328(\mathrm{~s}$, $\mathrm{N}^{+} \mathrm{O}^{-}$); 1,242 and 1,171 (m, Ar-F); Anal. Calc. for $\mathrm{C}_{14} \mathrm{H}_{7} \mathrm{FN}_{4} \mathrm{O}_{4}: \mathrm{C}: 53.51 \%$; $\mathrm{H}: 2.25 \%$; N: $17.83 \%$. Found: C: $53.06 \%$; H: $2.27 \%$; N: $17.86 \%$.

Furan-2-carboxylic acid (3-cyano-6,7-difluoro-1,4-di-N-oxidequinoxalin-2-yl)amide (12). Yield 66.1 \%; ${ }^{1} \mathrm{H}-\mathrm{NMR} \delta$ ppm: 11.50 (bs, $\left.1 \mathrm{H}, \mathrm{NH}\right) ; 8.65-8.58\left(\mathrm{~m}, 2 \mathrm{H}, \mathrm{H}_{8}+\mathrm{H}_{5}\right) ; 8.10$ (dd, $1 \mathrm{H}, \mathrm{H}_{5^{\prime}}, J_{5^{\prime}-4^{\prime}}=1.6 \mathrm{~Hz}$, $\left.J_{5^{\prime}-3^{\prime}}=0.6 \mathrm{~Hz}\right) ; 7.71\left(\mathrm{dd}, 1 \mathrm{H}, \mathrm{H}_{3^{\prime}}, J_{3^{\prime}-4^{\prime}}=3.7 \mathrm{~Hz}\right) ; 6.81\left(\mathrm{dd}, 1 \mathrm{H}, \mathrm{H}_{4^{\prime}}\right) ; \mathrm{IR} v \mathrm{~cm}^{-1}: 3,215(\mathrm{~m}, \mathrm{NH}) ; 2,236$ 
$(\mathrm{w}, \mathrm{C} \equiv \mathrm{N}) ; 1,689(\mathrm{~s}, \mathrm{C}=\mathrm{O}) ; 1,340\left(\mathrm{~s}, \mathrm{~N}^{+} \mathrm{O}^{-}\right) ; 1,270$ and 1,181 (m, Ar-F); Anal. Calc. for $\mathrm{C}_{14} \mathrm{H}_{6} \mathrm{~F}_{2} \mathrm{~N}_{4} \mathrm{O}_{4}$ : C: 50.61\%; H:1.82\%; N: 16.86\%. Found: C: 50.37\%; H: 1.98\%; N: 16.67\%.

Furan-2-carboxylic acid (3-cyano-1,4-di- $N$-oxide-7-trifluoromethylquinoxalin-2-yl)amide (13). Yield $78.1 \%$; ${ }^{1} \mathrm{H}-\mathrm{NMR} \delta$ ppm: 12.06 (bs, $\left.1 \mathrm{H}, \mathrm{NH}\right) ; 8.69$ (s, $\left.1 \mathrm{H}, \mathrm{H}_{8}\right) ; 8.64$ (d, $\left.1 \mathrm{H}, \mathrm{H}_{5}, J_{5-6}=9.0 \mathrm{~Hz}\right) ; 8.37$ (d, $\left.1 \mathrm{H}, \mathrm{H}_{6}\right) ; 8.07$ (d, $\left.1 \mathrm{H}, \mathrm{H}_{5^{\prime}}, J_{5^{\prime}-4^{\prime}}=1.7 \mathrm{~Hz}\right) ; 7.66\left(\mathrm{~d}, 1 \mathrm{H}, \mathrm{H}_{3^{\prime}}\right) ; 6.79\left(\mathrm{~d}, 1 \mathrm{H}, \mathrm{H}_{4^{\prime}}, J_{3^{\prime}-4^{\prime}}=3.5 \mathrm{~Hz}\right) ; \mathrm{IR} v \mathrm{~cm}^{-1}$ : 3,279 (m, NH); 2,244 (w, C $\equiv \mathrm{N}) ; 1,688(\mathrm{~s}, \mathrm{C}=\mathrm{O}) ; 1,325\left(\mathrm{~s}, \mathrm{~N}^{+} \mathrm{O}^{-}\right) ; 1,137\left(\mathrm{~s}, \mathrm{CF}_{3}\right)$; Anal. Calc. for $\mathrm{C}_{15} \mathrm{H}_{7} \mathrm{~F}_{3} \mathrm{~N}_{4} \mathrm{O}_{4}$ : C: 49.46\%; H: 1.96\%; N: 15.38\%. Found: C: 49.18\%; H: 2.02\%; N: 15.31\%.

Furan-2-carboxylic acid (3-cyano-1,4-di- $N$-oxide-6-trifluoromethylquinoxalin-2-yl)amide (14). Yield $31.1 \% ;{ }^{1} \mathrm{H}-\mathrm{NMR} \delta$ ppm: 12.06 (bs, $\left.1 \mathrm{H}, \mathrm{NH}\right) ; 8.72\left(\mathrm{~d}, 1 \mathrm{H}, \mathrm{H}_{5}, J_{5-7}=1.7 \mathrm{~Hz}\right) ; 8.66\left(\mathrm{~d}, 1 \mathrm{H}, \mathrm{H}_{8}, J_{8-7}=9.0\right.$ $\mathrm{Hz}) ; 8.30\left(\mathrm{dd}, 1 \mathrm{H}, \mathrm{H}_{7}, J_{7-8}=9.0 \mathrm{~Hz}, J_{7-5}=1.7 \mathrm{~Hz}\right) ; 8.09\left(\mathrm{dd}, 1 \mathrm{H}, \mathrm{H}_{5^{\prime}}, J_{5^{\prime}-4^{\prime}}=1.7 \mathrm{~Hz}, J_{5^{\prime}-3^{\prime}}=0.7 \mathrm{~Hz}\right)$; $7.69\left(\mathrm{dd}, 1 \mathrm{H}, \mathrm{H}_{3^{\prime}}, J_{3^{\prime}-4^{\prime}}=3.6 \mathrm{~Hz}\right) ; 6.80\left(\mathrm{dd}, 1 \mathrm{H}, \mathrm{H}_{4^{\prime}}\right) ; \mathrm{IR} v \mathrm{~cm}^{-1}: 3,263(\mathrm{~m}, \mathrm{NH}) ; 2,232(\mathrm{w}, \mathrm{C} \equiv \mathrm{N}) ; 1,701$ $(\mathrm{s}, \mathrm{C}=\mathrm{O}) ; 1,341\left(\mathrm{~s}, \mathrm{~N}^{+} \mathrm{O}^{-}\right) ; 1,132\left(\mathrm{~s}, \mathrm{CF}_{3}\right)$; Anal. Calc. for $\mathrm{C}_{15} \mathrm{H}_{7} \mathrm{~F}_{3} \mathrm{~N}_{4} \mathrm{O}_{4}: \mathrm{C}: 49.46 \% ; \mathrm{H}: 1.96 \%$; $\mathrm{N}$ 15.38\%. Found: C: 49.59\%; H: 1.89\%; N: 15.09\%.

Thiophene-2-carboxylic acid (3-cyano-1,4-di- $N$-oxidequinoxalin-2-yl)amide (15). Yield $33.3 \%$; ${ }^{1} \mathrm{H}$ NMR $\delta$ ppm: $11.65(\mathrm{bs}, 1 \mathrm{H}, \mathrm{NH}) ; 8.54\left(\mathrm{dd}, 1 \mathrm{H}, \mathrm{H}_{8}, J_{8-7}=8.6 \mathrm{~Hz}, J_{8-6}=0.7 \mathrm{~Hz}\right) ; 8.50\left(\mathrm{dd}, 1 \mathrm{H}, \mathrm{H}_{5}, J_{5-6}\right.$ $\left.=8.6 \mathrm{~Hz}, J_{5-7}=0.8 \mathrm{~Hz}\right) ; 8.30\left(\mathrm{dd}, 1 \mathrm{H}, \mathrm{H}_{5}, J_{5^{\prime}-4^{\prime}}=3.7 \mathrm{~Hz}, J_{5^{\prime}-3}=0.9 \mathrm{~Hz}\right) ; 8.12\left(\mathrm{td}, 1 \mathrm{H}, J_{6-7}=7.1 \mathrm{~Hz}\right.$, H6); 8.10-8.00 (m, 2H, $\left.\mathrm{H}_{7}, \mathrm{H}_{3^{\prime}}\right)$; 7.32 (dd, $\left.1 \mathrm{H}, \mathrm{H}_{4^{\prime}}, J_{4^{\prime}-3}{ }^{\prime}=4.9 \mathrm{~Hz}, J_{4^{\prime}-5^{\prime}}=3.9 \mathrm{~Hz}\right) ; \mathrm{IR} v \mathrm{~cm}^{-1}: 3,231$ (m, $\mathrm{NH}) ; 2,232(\mathrm{w}, \mathrm{C} \equiv \mathrm{N}) ; 1,681(\mathrm{~s}, \mathrm{C}=\mathrm{O}) ; 1,326\left(\mathrm{~s}, \mathrm{~N}^{+} \mathrm{O}^{-}\right)$; Anal. Calc. for $\mathrm{C}_{14} \mathrm{H}_{8} \mathrm{~N}_{4} \mathrm{O}_{3} \mathrm{~S}: \mathrm{C}: 53.85 \%$; $\mathrm{H}$ : $2.56 \%$; N: 17.95\%. Found: C: 53.88\%; H: 2.82\%; N: 18.35\%.

Thiophene-2-carboxylic acid (3-cyano-1,4-di- $\mathrm{N}$-oxide-6-methylquinoxalin-2-yl)amide (16). Yield 1.2 $\% ;{ }^{1} \mathrm{H}-\mathrm{NMR} \delta$ ppm: 11.62 (bs, $\left.1 \mathrm{H}, \mathrm{NH}\right) ; 8.39\left(\mathrm{~d}, \mathrm{H}_{8}, 1 \mathrm{H}, J_{8-7}=8.8 \mathrm{~Hz}\right) ; 8.35\left(\mathrm{~s}, 1 \mathrm{H}, \mathrm{H}_{5}\right) ; 8.30(\mathrm{~m}, 1 \mathrm{H}$, $\left.\mathrm{H}_{5}{ }^{\prime}\right) ; 8.05\left(\mathrm{~d}, 1 \mathrm{H}, \mathrm{H}_{3}{ }^{\prime}, J_{3^{\prime}-4}\right.$, = $\left.4.9 \mathrm{~Hz}\right) ; 7.88\left(\mathrm{~d}, 1 \mathrm{H}, \mathrm{H}_{7}\right) ; 7.33-7.31\left(\mathrm{~m}, 1 \mathrm{H}, \mathrm{H}_{4}{ }^{\prime}\right) ; 2.66\left(\mathrm{~s}, 3 \mathrm{H}, \mathrm{CH}_{3}\right) ; \mathrm{IR} v$ $\mathrm{cm}^{-1}$ : 3,276(w, NH); $2,225(\mathrm{w}, \mathrm{C} \equiv \mathrm{N}) ; 1,664(\mathrm{~s}, \mathrm{C}=\mathrm{O}) ; 1,329\left(\mathrm{~s}, \mathrm{~N}^{+} \mathrm{O}^{-}\right)$; Anal. Calc. for $\mathrm{C}_{15} \mathrm{H}_{10} \mathrm{~N}_{4} \mathrm{O}_{3} \mathrm{~S}$ : C: $55.22 \%$; H: $3.07 \%$; N: 17.18\%. Found: C: $55.01 \%$; H: $3.17 \%$; N: $17.39 \%$.

Thiophene-2-carboxylic acid (3-cyano-6,7-dimethyl-1,4-di-N-oxidequinoxalin-2-yl)amide (17). Yield $1.4 \%$; ${ }^{1} \mathrm{H}-\mathrm{NMR} \delta$ ppm: 11.59 (bs, $\left.1 \mathrm{H}, \mathrm{NH}\right) ; 8.32$ (s, $1 \mathrm{H}, \mathrm{H}_{5}$ ); 8.28 (s, 2H, $\left.\mathrm{H}_{8}+\mathrm{H}_{5}\right) ; 8.03\left(\mathrm{~s}, 1 \mathrm{H}, \mathrm{H}_{3^{\prime}}\right.$ ); $7.31\left(\mathrm{~s}, 1 \mathrm{H}, \mathrm{H}_{4}\right)$ ); 2.6-2.4 (m, 6H, 2xCH$) ; \mathrm{IR} v \mathrm{~cm}^{-1}: 3,206(\mathrm{~m}, \mathrm{NH}) ; 2,225(\mathrm{w}, \mathrm{C} \equiv \mathrm{N}) ; 1,667(\mathrm{~s}, \mathrm{C}=\mathrm{O})$; $1,327\left(\mathrm{~s}, \mathrm{~N}^{+} \mathrm{O}^{-}\right)$; Anal. Calc. for $\mathrm{C}_{16} \mathrm{H}_{12} \mathrm{~N}_{4} \mathrm{O}_{3} \mathrm{~S}: \mathrm{C}: 56.47 \%$; H: 3.53\%; N: 16.47\%. Found: C: $56.72 \%$; H: $3.64 \%$; N: $16.72 \%$.

Thiophene-2-carboxylic acid (3-cyano-1,4-di-N-oxide-6-methoxyquinoxalin-2-yl)amide (18). Yield $41.1 \% ;{ }^{1} \mathrm{H}-\mathrm{NMR} \delta$ ppm: 11.53 (bs, $\left.1 \mathrm{H}, \mathrm{NH}\right) ; 8.46$ (d, $\left.1 \mathrm{H}, \mathrm{H}_{8}, J_{8-7}=9.4 \mathrm{~Hz}\right) ; 8.28$ (dd, 1H, $\mathrm{H}_{5^{\prime}}, J_{5^{\prime}-4}{ }^{\prime}=$ $\left.3.8 \mathrm{~Hz}, J_{5^{\prime}-3^{\prime}}=0.9 \mathrm{~Hz}\right) ; 8.05\left(\mathrm{dd}, 1 \mathrm{H}, \mathrm{H}_{3}{ }^{\prime}, J_{3^{\prime}-4}=3.8 \mathrm{~Hz}\right) ; 7.79\left(\mathrm{~d}, 1 \mathrm{H}, \mathrm{H}_{5}, J_{5-7}=2.7 \mathrm{~Hz}\right) ; 7.73(\mathrm{dd}, 1 \mathrm{H}$, $\left.\mathrm{H}_{7}\right) ; 7.32\left(\mathrm{dd}, 1 \mathrm{H}, \mathrm{H}_{4}\right) ; 4.03\left(\mathrm{~s}, 3 \mathrm{H}, \mathrm{OCH}_{3}\right) ; \mathrm{IR} v \mathrm{~cm}^{-1}: 3,276(\mathrm{~m}, \mathrm{NH}) ; 2,232(\mathrm{w}, \mathrm{C} \equiv \mathrm{N}) ; 1,673(\mathrm{~s}$, $\mathrm{C}=\mathrm{O}) ; 1,320\left(\mathrm{~s}, \mathrm{~N}^{+} \mathrm{O}^{-}\right)$; Anal. Calc. for $\mathrm{C}_{15} \mathrm{H}_{10} \mathrm{~N}_{4} \mathrm{O}_{4} \mathrm{~S}: \mathrm{C}: 52.63 \%$; $\mathrm{H}: 2.93 \%$; N: 16.37\%. Found: C: 52.49\%; H: $3.06 \%$; N: $16.35 \%$. 
Thiophene-2-carboxylic acid (6-chloro-3-cyano-1,4-di-N-oxidequinoxalin-2-yl)amide (19). Yield 33.2 $\%$; ${ }^{1} \mathrm{H}-\mathrm{NMR} \delta$ ppm: 11.66 (bs, $\left.1 \mathrm{H}, \mathrm{NH}\right) ; 8.54-8.48\left(\mathrm{~m}, 2 \mathrm{H}, \mathrm{H}_{8}+\mathrm{H}_{5}\right) ; 8.30\left(\mathrm{dd}, 1 \mathrm{H}, \mathrm{H}_{5}, J_{5}{ }^{\prime}-4,=3.8 \mathrm{~Hz}\right.$, $\left.J_{5^{\prime}-3},=0.8 \mathrm{~Hz}\right) ; 8.15\left(\mathrm{dd}, 1 \mathrm{H}, \mathrm{H}_{7}, J_{7-8}=9.30 \mathrm{~Hz}, J_{7-5}=2.2 \mathrm{~Hz}\right.$,); $8.06\left(\mathrm{dd}, 1 \mathrm{H}, \mathrm{H}_{3}, J_{3^{\prime}-4},=5.0 \mathrm{~Hz}\right) ; 7.32$ $\left(\mathrm{t}, 1 \mathrm{H}, \mathrm{H}_{4}\right)$; IR $v \mathrm{~cm}^{-1}: 3,295(\mathrm{~m}, \mathrm{NH}) ; 2,251(\mathrm{w}, \mathrm{C} \equiv \mathrm{N}) ; 1,671(\mathrm{~s}, \mathrm{C}=\mathrm{O}) ; 1,323\left(\mathrm{~s}, \mathrm{~N}^{+} \mathrm{O}^{-}\right)$; Anal. Calc. for $\mathrm{C}_{14} \mathrm{H}_{7} \mathrm{ClN}_{4} \mathrm{O}_{3} \mathrm{~S}$ : C: 48.48\%; H: 2.02\%; N: 16.16\%. Found: C: 48.58\%; H: 2.16\%; N: 16.15\%.

Thiophene-2-carboxylic acid (3-cyano-6,7-dichloro-1,4-di- $N$-oxidequinoxalin-2-yl)amide (20). Yield $19.4 \%$; ${ }^{1} \mathrm{H}-\mathrm{NMR} \delta$ ppm: $8.66\left(\mathrm{~s}, 1 \mathrm{H}, \mathrm{H}_{8}\right) ; 8.64\left(\mathrm{~s}, 1 \mathrm{H}, \mathrm{H}_{5}\right) ; 8.13\left(\mathrm{~s}, 1 \mathrm{H}, \mathrm{H}_{5}\right.$ ) $; 7.96\left(\mathrm{~d}, 1 \mathrm{H}, \mathrm{H}_{3^{\prime},}, J_{3^{\prime}-4^{\prime}}=\right.$

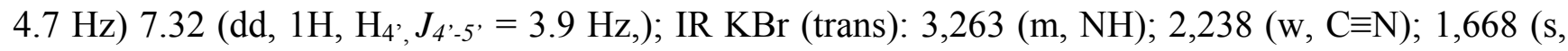
$\mathrm{C}=\mathrm{O}) ; 1,335\left(\mathrm{~s}, \mathrm{~N}^{+} \mathrm{O}^{-}\right)$; Anal. Calc. for $\mathrm{C}_{14} \mathrm{H}_{6} \mathrm{Cl}_{2} \mathrm{~N}_{4} \mathrm{O}_{3} \mathrm{~S}: \mathrm{C}: 44.09 \%$; H: 1.57\%; N: 14.70\%. Found: C: $43.83 \%$; H: $1.67 \%$; N: $14.70 \%$.

Thiophene-2-carboxylic acid (3-cyano-1,4-di-N-oxide-6-fluoroquinoxalin-2-yl)amide (21). Yield 46.0 $\% ;{ }^{1} \mathrm{H}-\mathrm{NMR} \delta$ ppm: 11.63 (bs, $\left.1 \mathrm{H}, \mathrm{NH}\right) ; 8.60\left(\mathrm{dd}, 1 \mathrm{H}, \mathrm{H}_{8}, J_{8-7}=9.5 \mathrm{~Hz}, J_{8-F}=4.9 \mathrm{~Hz}\right.$ ); 8.31-8.27 (m, $\left.2 \mathrm{H}, \mathrm{H}_{5}+\mathrm{H}_{5^{\prime}}\right) ; 8.06-8.01\left(\mathrm{~m}, 2 \mathrm{H}, \mathrm{H}_{7}+\mathrm{H}_{3^{\prime}}\right) ; 7.32\left(\mathrm{t}, 1 \mathrm{H}, \mathrm{H}_{4}\right)$ ); IR $v \mathrm{~cm}^{-1}: 3,263(\mathrm{~m}, \mathrm{NH}) ; 2,226(\mathrm{w}, \mathrm{C} \equiv \mathrm{N})$; $1,665(\mathrm{~s}, \mathrm{C}=\mathrm{O}) ; 1,327\left(\mathrm{~s}, \mathrm{~N}^{+} \mathrm{O}^{-}\right)$; Anal. Calc. for $\mathrm{C}_{14} \mathrm{H}_{7} \mathrm{FN}_{4} \mathrm{O}_{3} \mathrm{~S}: \mathrm{C}: 50.91 \%$; $\mathrm{H}: 2.12 \%$; N: $16.97 \%$. Found: C: $51.18 \%$; H: 2.24\%; N: 17.06\%.

Thiophene-2-carboxylic acid (3-cyano-6,7-difluoro-1,4-di- $N$-oxidequinoxalin-2-yl)amide (22). Yield $69.1 \% ;{ }^{1} \mathrm{H}-\mathrm{NMR} \delta$ ppm: 11.74 (bs, 1H, NH); 8.70-8.50 (m, 2H, $\left.\mathrm{H}_{8}+\mathrm{H}_{5}\right) ; 8.25-8.35\left(\mathrm{~m}, 1 \mathrm{H}, \mathrm{H}_{5}\right.$ ); 8.07 $\left(\mathrm{d}, 1 \mathrm{H}, \mathrm{H}_{3^{\prime}}, J_{3^{\prime}-4^{\prime}}=4.9 \mathrm{~Hz}\right) ; 7.34-7.31\left(\mathrm{~m}, 1 \mathrm{H}, \mathrm{H}_{4}\right)$; IR $v \mathrm{~cm}^{-1}: 3,238(\mathrm{~m}, \mathrm{NH}) ; 2,238(\mathrm{w}, \mathrm{C} \equiv \mathrm{N}) ; 1,673$ (s, $\mathrm{C}=\mathrm{O}) ; 1,341\left(\mathrm{~s}, \mathrm{~N}^{+} \mathrm{O}^{-}\right)$; Anal. Calc. for $\mathrm{C}_{14} \mathrm{H}_{6} \mathrm{~F}_{2} \mathrm{~N}_{4} \mathrm{O}_{3} \mathrm{~S}: \mathrm{C}: 48.27 \%$; $\mathrm{H}: 1.72 \%$; N: 16.01\%. Found: C: $48.47 \% ; \mathrm{H}: 1.80 \%$; N: $16.18 \%$.

Thiophene-2-carboxylic acid (3-cyano-7-trifluoromethyl-1,4-di- $\mathrm{N}$-oxidequinoxalin-2-yl)amide (23). Yield $11.7 \%$; ${ }^{1} \mathrm{H}-\mathrm{NMR} \delta$ ppm: 11.85 (bs, $\left.1 \mathrm{H}, \mathrm{NH}\right) ; 8.75\left(\mathrm{~d}, 1 \mathrm{H}, \mathrm{H}_{8}, J_{8-6}=0.75 \mathrm{~Hz}\right) ; 8.65\left(\mathrm{~d}, 1 \mathrm{H}, \mathrm{H}_{5}\right.$, $\left.J_{5-6}=9.0 \mathrm{~Hz}\right) ; 8.39\left(\mathrm{dd}, 1 \mathrm{H}, \mathrm{H}_{6}, J_{6-5}=9.1 \mathrm{~Hz}, J_{6-8}=1.8 \mathrm{~Hz}\right) ; 8.27\left(\mathrm{dd}, 1 \mathrm{H}, \mathrm{H}_{5}, J_{5^{\prime}-4},=3.8 \mathrm{~Hz}, J_{5^{\prime}-3}{ }^{\prime}=\right.$ $1.1 \mathrm{~Hz}) ; 8.05\left(\mathrm{dd}, 1 \mathrm{H}, \mathrm{H}_{3^{\prime}}, J_{3^{\prime}-4}{ }^{\prime}=5.0 \mathrm{~Hz}\right) ; 7.31\left(\mathrm{dd}, 1 \mathrm{H}, \mathrm{H}_{4}\right.$ ); IR KBr (trans): 3,263 (w, NH); 2,238 (w, $\mathrm{C} \equiv \mathrm{N}) ; 1,668(\mathrm{~s}, \mathrm{C}=\mathrm{O}) ; 1,335\left(\mathrm{~s}, \mathrm{~N}^{+} \mathrm{O}^{-}\right)$; Anal. Calc. for $\mathrm{C}_{15} \mathrm{H}_{7} \mathrm{~F}_{3} \mathrm{~N}_{4} \mathrm{O}_{3} \mathrm{~S}: \mathrm{C}: 47.37 \% ; \mathrm{H}: 1.84 \%$; : 14.74\%. Found: C: 47.67\%; H: 1.77\%; N: 14.80\%.

Thiophene-2-carboxylic acid (3-cyano-6-trifluoromethyl-1,4-di- $\mathrm{N}$-oxidequinoxalin-2-yl)amide (24). Yield $12.8 \%$; ${ }^{1} \mathrm{H}-\mathrm{NMR} \delta$ ppm: 11.83 (bs, $\left.1 \mathrm{H}, \mathrm{NH}\right) ; 8.74$ (s, 1H, $\left.\mathrm{H}_{5}\right) ; 8.68$ (d, 1H, $\mathrm{H}_{8}, J_{8-7}=9.24 \mathrm{~Hz}$ ); 8.35-8.28 (m, $\left.2 \mathrm{H}, \mathrm{H}_{7}, \mathrm{H}_{5}{ }^{\prime}\right) ; 8.06\left(\mathrm{dd}, 1 \mathrm{H}, \mathrm{H}_{3^{\prime}}, J_{3^{\prime}-4}{ }^{\prime}=5.0 \mathrm{~Hz}, J_{3^{\prime}-5}{ }^{\prime}=1.0 \mathrm{~Hz}\right) ; 7.33-7.31\left(\mathrm{~m}, 1 \mathrm{H}, \mathrm{H}_{4}\right)^{\prime}$; IR $\mathrm{KBr}$ (trans): 3,270 (w, NH); 2,226 (w, C $\equiv \mathrm{N}) ; 1,679(\mathrm{~s}, \mathrm{C}=\mathrm{O}) ; 1,339\left(\mathrm{~s}, \mathrm{~N}^{+} \mathrm{O}^{-}\right)$; Anal. Calc. for $\mathrm{C}_{15} \mathrm{H}_{7} \mathrm{~F}_{3} \mathrm{~N}_{4} \mathrm{O}_{3} \mathrm{~S}: \mathrm{C}: 47.37 \%$; $\mathrm{H}: 1.84 \%$; $: 14.74 \%$. Found: $\mathrm{C}: 47.11 \% ; \mathrm{H}: 1.74 \%$; N: $14.64 \%$.

General procedure for the synthesis of 5-nitrofuran-2-carboxylic acid (3-cyano-1,4-di- $N$-oxidequinoxalin-2-yl)amide derivatives and 5-nitrothiophene-2-carboxylic acid (3-cyano-1,4-N-oxidequinoxalin-2-yl)amide derivatives 25-44

To a solution of the corresponding 3-amino-1,4-di- $N$-oxide-quinoxaline-2-carbonitrile II ( 2 mmol) in DMF $(5 \mathrm{~mL})$ are added 5-nitrothiophene-2-carboxylic acid or 5-nitrofuran-2-carboxylic acid (3 $\mathrm{mmol}$ ). The reaction mixture is then gently stirred at room temperature under anhydrous conditions. 
Continuing with the synthesis, $N$-(3-dimethylaminopropyl)- $N$ '-ethylcarbodiimide hydrochloride (EDCI, $6 \mathrm{mmol}$ ) are added, and the color changes to dark-red. When the dissolution is completed, 4dimethyl-aminopyridine (DMAP, 6 or $7 \mathrm{mmol}$ ) are added. The reaction mixture is stirred between 17 $\mathrm{h}-72 \mathrm{~h}$. After that, EtOAc $(200 \mathrm{~mL})$ is added and the organic phase is extracted, first with $10 \% \mathrm{HCl}$ and then with saturated $\mathrm{NaHCO}_{3}$. The basic phase is treated with $\mathrm{HCl} 37 \%$ until $\mathrm{pH} 2$, usually shown by the color changing to yellow. This phase is extracted with dichloromethane $(3 \times 75 \mathrm{~mL})$, dried with anhydrous $\mathrm{Na}_{2} \mathrm{SO}_{4}$ and filtered. The solvent is removed in vacuo. The resulting residue is precipitated with diethyl ether and filtered in order to obtain a yellow-orange solid. Sometimes, when DMAP is added, the compound precipitates due to its acidity. In those cases, $\mathrm{HCl}(300 \mathrm{~mL})$ is added and the mixture is stirred gently. The 3-amino-1,4-di- $N$-oxide-quinoxaline-2-carbonitrile derivatives are dissolved, and the precipitated compound is filtered. The compound is first washed with $10 \% \mathrm{HCl}$ and then with diethyl ether.

5-Nitrofuran-2-carboxylic acid (3-cyano-1,4-di-N-oxide-quinoxalin-2-yl)-amide (25). Yield 27.8\%; ${ }^{1} \mathrm{H}-\mathrm{NMR} \delta$ ppm: 8.52-8.48 (m, 2H, $\left.\mathrm{H}_{8}+\mathrm{H}_{5}\right)$; 8.14-8.10 (m, 1H, $\left.\mathrm{H}_{7}\right)$; 8.06-8.02 (m,1H, $\left.\mathrm{H}_{6}\right) ; 7.91(\mathrm{~d}, 1 \mathrm{H}$, $\left.\mathrm{H}_{4^{\prime}}, J_{4^{\prime}-3^{\prime}}=3.9 \mathrm{~Hz}\right) ; 7.85\left(\mathrm{~d}, 1 \mathrm{H}, \mathrm{H}_{3^{\prime}}\right)$; IR $v \mathrm{~cm}^{-1}: 3,263(\mathrm{~m}, \mathrm{NH}) ; 2,232(\mathrm{w}, \mathrm{C} \equiv \mathrm{N}) ; 1,694(\mathrm{~s}, \mathrm{C}=\mathrm{O})$; $1,533\left(\mathrm{~s}, \mathrm{NO}_{2}\right) ; 1,357\left(\mathrm{~s}, \mathrm{NO}_{2}\right) ; 1,333\left(\mathrm{~s}, \mathrm{~N}^{+} \mathrm{O}^{-}\right)$; Anal. Calc. for $\mathrm{C}_{14} \mathrm{H}_{7} \mathrm{~N}_{5} \mathrm{O}_{6}: \mathrm{C}: 49.28 \% ; \mathrm{H}: 2.07 \%$; $\mathrm{N}: 20.52 \%$. Found: C: $48.88 \%$; H: 2.24\%; N: $20.70 \%$.

5-Nitrofuran-2-carboxylic acid (3-cyano-6-methyl-1,4-di-N-oxide-quinoxalin-2-yl)amide (26). Yield $23.5 \%$; ${ }^{1} \mathrm{H}-\mathrm{NMR} \delta$ ppm: 8.41 (d, $\left.1 \mathrm{H}, \mathrm{H}_{8}, J_{8-7}=8.9 \mathrm{~Hz}\right) ; 8.31\left(\mathrm{~s}, 1 \mathrm{H}, \mathrm{H}_{5}\right) ; 7.95$ (d, 1H, $\left.\mathrm{H}_{7}\right) ; 7.89$ (d, $\left.1 \mathrm{H}, \mathrm{H}_{4}, J_{4^{\prime}-3^{\prime}}=3.8 \mathrm{~Hz}\right) ; 7.84\left(\mathrm{~d}, 1 \mathrm{H}, \mathrm{H}_{3^{\prime}}\right) ; 2.61\left(\mathrm{~s}, 3 \mathrm{H}, \mathrm{CH}_{3}\right) ; \mathrm{IR} v \mathrm{~cm}^{-1}: 3,250(\mathrm{w}, \mathrm{NH}) ; 2,236(\mathrm{w}$, $\mathrm{C} \equiv \mathrm{N}) ; 1,701(\mathrm{~s}, \mathrm{C}=\mathrm{O}) ; 1,527\left(\mathrm{~s}, \mathrm{NO}_{2}\right) ; 1,355\left(\mathrm{~s}, \mathrm{NO}_{2}\right) ; 1,332\left(\mathrm{~s}, \mathrm{~N}^{+} \mathrm{O}^{-}\right)$; Anal. Calc. for $\mathrm{C}_{15} \mathrm{H}_{9} \mathrm{~N}_{5} \mathrm{O}_{6}: \mathrm{C}$ : 50.71\%; H: 2.55\%; N: 19.71\%. Found: C: 50.78\%; H: 2.61\%; N: 19.83\%.

5-Nitrofuran-2-carboxylic acid (3-cyano-6,7-dimethyl-1,4-di-N-oxide-quinoxalin-2-yl)amide (27). Yield $11.3 \%$; ${ }^{1} \mathrm{H}-\mathrm{NMR} \delta$ ppm: $8.31\left(\mathrm{~s}, 1 \mathrm{H}, \mathrm{H}_{8}\right) ; 8.28\left(\mathrm{~s}, 1 \mathrm{H}, \mathrm{H}_{5}\right) ; 7.91\left(\mathrm{~d}, 1 \mathrm{H}, \mathrm{H}_{4}, J_{4^{\prime}-3}{ }^{\prime}=3.9 \mathrm{~Hz}\right)$; $7.85\left(\mathrm{~d}, 1 \mathrm{H}, \mathrm{H}_{3}\right) ; 2.54\left(\mathrm{~s}, 3 \mathrm{H}, \mathrm{CH}_{3}\right) ; 2.53\left(\mathrm{~s}, 3 \mathrm{H}, \mathrm{CH}_{3}\right) ; \mathrm{IR} v \mathrm{~cm}^{-1}: 3,173(\mathrm{~m}, \mathrm{NH}) ; 2,942\left(\mathrm{w}, \mathrm{CH}_{3}\right)$; $2,232(\mathrm{w}, \mathrm{C} \equiv \mathrm{N}) ; 1,700(\mathrm{~s}, \mathrm{C}=\mathrm{O}) ; 1,542\left(\mathrm{~s}, \mathrm{NO}_{2}\right) ; 1,349\left(\mathrm{~s}, \mathrm{NO}_{2}\right) ; 1,317\left(\mathrm{~s}, \mathrm{~N}^{+} \mathrm{O}^{-}\right)$; Anal. Calc. for $\mathrm{C}_{16} \mathrm{H}_{11} \mathrm{~N}_{5} \mathrm{O}_{6}$ : C: 52.04\%; H: 3.00\%; N: 18.96\%. Found: C: 52.35\%; H: 3.25\%; N: 18.61\%.

5-Nitrofuran-2-carboxylic acid (3-cyano-6-methoxy-1,4-di- $\mathrm{N}$-oxide-quinoxalin-2-yl)amide (28). Yield $30.1 \% ;{ }^{1} \mathrm{H}-\mathrm{NMR} \delta$ ppm: $8.45\left(\mathrm{~d}, 1 \mathrm{H}, \mathrm{H}_{8}, J_{8-7}=9.0 \mathrm{~Hz}\right) ; 7.90\left(\mathrm{~d}, 1 \mathrm{H}, \mathrm{H}_{4^{\prime}}, J_{4^{\prime}-3^{\prime}}=3.9 \mathrm{~Hz}\right) ; 7.85(\mathrm{~d}, 1 \mathrm{H}$, $\left.H_{3^{\prime}}\right) ; 7.79\left(\mathrm{~d}, 1 \mathrm{H}, \mathrm{H}_{5}, J_{5-7}=2.5 \mathrm{~Hz}\right) ; 7.74\left(\mathrm{dd}, 1 \mathrm{H}, \mathrm{H}_{7}\right) ; 4.04\left(\mathrm{~s}, 3 \mathrm{H}, \mathrm{OCH}_{3}\right) ; \mathrm{IR} v \mathrm{~cm}^{-1}: 3,129(\mathrm{~m}, \mathrm{NH})$; $2,239(\mathrm{w}, \mathrm{C} \equiv \mathrm{N}) ; 1,693(\mathrm{~s}, \mathrm{C}=\mathrm{O}) ; 1,538\left(\mathrm{~s}, \mathrm{NO}_{2}\right) ; 1,388\left(\mathrm{~s}, \mathrm{NO}_{2}\right) ; 1,326\left(\mathrm{~s}, \mathrm{~N}^{+} \mathrm{O}^{-}\right)$; Anal. Calc. for $\mathrm{C}_{15} \mathrm{H}_{9} \mathrm{~N}_{5} \mathrm{O}_{7}$ : C: $48.53 \%$; H: $2.44 \%$; N: 18.86\%. Found: C: $48.23 \%$; H: $2.41 \%$; N: $18.66 \%$.

5-Nitrofuran-2-carboxylic acid (6-chloro-3-cyano-1,4-di- $N$-oxide-quinoxalin-2-yl)amide (29). Yield $16.5 \%$; ${ }^{1} \mathrm{H}-\mathrm{NMR} \delta$ ppm: 8.49-8.45 (m, $\left.2 \mathrm{H}, \mathrm{H}_{8}+\mathrm{H}_{5}\right) ; 8.13\left(\mathrm{dd}, 1 \mathrm{H}, \mathrm{H}_{7}, J_{7-8}=9.2 \mathrm{~Hz}, J_{7-5}=2.1 \mathrm{~Hz}\right) ; 7.87$ $\left(\mathrm{d}, 1 \mathrm{H}, \mathrm{H}_{4^{\prime}}, J_{4^{\prime}-3^{\prime}}=3.8 \mathrm{~Hz}\right) ; 7.83\left(\mathrm{~d}, 1 \mathrm{H}, \mathrm{H}_{3^{\prime}}\right) ; 5.75(\mathrm{~s}, 1 \mathrm{H}, \mathrm{NH}) ; \mathrm{IR} v_{\mathrm{cm}}^{-1}: 3,273(\mathrm{~m}, \mathrm{NH}) ; 2,238$ (w, $\mathrm{C} \equiv \mathrm{N}) ; 1,711(\mathrm{~s}, \mathrm{C}=\mathrm{O}) ; 1,537\left(\mathrm{~s}, \mathrm{NO}_{2}\right) ; 1,351\left(\mathrm{~s}, \mathrm{NO}_{2}\right) ; 1,327\left(\mathrm{~s}, \mathrm{~N}^{+} \mathrm{O}^{-}\right) ; 1,077$ (m, Ar-Cl); Anal. Calc. for $\mathrm{C}_{14} \mathrm{H}_{6} \mathrm{ClN}_{5} \mathrm{O}_{6}$ : C: $44.76 \%$; $\mathrm{H}: 1.61 \%$; N: 18.64\%. Found: C: 44.72\%; H: 1.80\%; N: 18.53\%. 
5-Nitrofuran-2-carboxylic acid (6,7-dichloro-3-cyano-1,4-di- $N$-oxide-quinoxalin-2-yl)amide (30). Yield $12.4 \%$; ${ }^{1} \mathrm{H}-\mathrm{NMR} \delta$ ppm: $8.70\left(\mathrm{~s}, 1 \mathrm{H}, \mathrm{H}_{8}\right) ; 8.66\left(\mathrm{~s}, 1 \mathrm{H}, \mathrm{H}_{5}\right) ; 7.87\left(\mathrm{~d}, 1 \mathrm{H}, \mathrm{H}_{4}, J_{4^{\prime}-3}{ }^{\prime}=3.9 \mathrm{~Hz}\right)$; $7.84\left(\mathrm{~d}, 1 \mathrm{H}, \mathrm{H}_{3}\right)$ ); IR $v \mathrm{~cm}^{-1}: 3,263(\mathrm{~m}, \mathrm{NH}) ; 2,244(\mathrm{w}, \mathrm{C} \equiv \mathrm{N}) ; 1,711(\mathrm{~s}, \mathrm{C}=\mathrm{O}) ; 1,539\left(\mathrm{~s}, \mathrm{NO}_{2}\right) ; 1,355(\mathrm{~s}$, $\left.\mathrm{NO}_{2}\right) ; 1,330\left(\mathrm{~s}, \mathrm{~N}^{+} \mathrm{O}^{-}\right) ; 1,073(\mathrm{~m}, \mathrm{Ar}-\mathrm{Cl})$; Anal. Calc. for $\mathrm{C}_{14} \mathrm{H}_{5} \mathrm{Cl}_{2} \mathrm{~N}_{5} \mathrm{O}_{6}: \mathrm{C}: 41.00 \% ; \mathrm{H}: 1.23 \%$; 17.08\%. Found: C: 41.15\%; H: 1.30\%; N: 17.25\%.

5-Nitrofuran-2-carboxylic acid (6-fluoro-3-cyano-1,4-di-N-oxide-quinoxalin-2-yl)amide (31). Yield $9.9 \%$; ${ }^{1} \mathrm{H}-\mathrm{NMR} \delta$ ppm: $8.58\left(\mathrm{dd}, 1 \mathrm{H}, \mathrm{H}_{8}, J_{8-7}=9.4 \mathrm{~Hz}, J_{8-\mathrm{F}}=5.0 \mathrm{~Hz}\right) ; 8.30\left(\mathrm{dd}, 1 \mathrm{H}, \mathrm{H}_{5}, J_{5-\mathrm{F}}=8.7 \mathrm{~Hz}\right.$, $\left.J_{5-7}=2.5 \mathrm{~Hz}\right) ; 8.06-8.01\left(\mathrm{~m}, 1 \mathrm{H}, \mathrm{H}_{7}\right) ; 7.90\left(\mathrm{~d}, 1 \mathrm{H}, \mathrm{H}_{4}, J_{4^{\prime}-3}{ }^{\prime}=3.9 \mathrm{~Hz}\right) ; 7.84\left(\mathrm{~d}, 1 \mathrm{H}, \mathrm{H}_{3}{ }^{\prime}\right) ; \mathrm{IR} v \mathrm{~cm}^{-1}$ : $3,237(\mathrm{~m}, \mathrm{NH}) ; 2,238(\mathrm{w}, \mathrm{C} \equiv \mathrm{N}) ; 1,708(\mathrm{~s}, \mathrm{C}=\mathrm{O}) ; 1,536\left(\mathrm{~s}, \mathrm{NO}_{2}\right) ; 1,357\left(\mathrm{~s}, \mathrm{NO}_{2}\right) ; 1,331\left(\mathrm{~s}, \mathrm{~N}^{+} \mathrm{O}^{-}\right)$; 1,114 (m, Ar-F); Anal. Calc. for $\mathrm{C}_{14} \mathrm{H}_{6} \mathrm{FN}_{5} \mathrm{O}_{6}: \mathrm{C}: 46.81 \%$; $\mathrm{H}: 1.68 \%$; N: 19.50\% Found: C: 46.53\%; H: $1.74 \% ; \mathrm{N}: 19.55 \%$.

5-Nitrofuran-2-carboxylic acid (6,7-difluoro-3-cyano-1,4-di-N-oxide-quinoxalin-2-yl)amide (32). Yield $38.6 \%$; ${ }^{1} \mathrm{H}-\mathrm{NMR} \delta$ ppm: $8.63\left(\mathrm{dd}, 1 \mathrm{H}, \mathrm{H}_{8}, J_{8-\mathrm{F} 7}=9.9 \mathrm{~Hz}, J_{8-\mathrm{F} 6}=7.2 \mathrm{~Hz}\right) ; 8.58\left(\mathrm{dd}, 1 \mathrm{H}, \mathrm{H}_{5}, J_{5-\mathrm{F} 6}\right.$ $\left.=9.9 \mathrm{~Hz}, J_{5-\mathrm{F} 7}=7.3 \mathrm{~Hz}\right) ; 7.89\left(\mathrm{~d}, 1 \mathrm{H}, \mathrm{H}_{4}, J_{4^{\prime}-3^{\prime}}=3.9 \mathrm{~Hz}\right) ; 7.84\left(\mathrm{~d}, 1 \mathrm{H}, \mathrm{H}_{3}{ }^{\prime}\right) ; 5.76(\mathrm{~s}, 1 \mathrm{H}, \mathrm{NH}) ; \mathrm{IR} \mathrm{v} \mathrm{cm}^{-}$ ${ }^{1}$ : $3,268(\mathrm{~m}, \mathrm{NH}) ; 2,244(\mathrm{w}, \mathrm{C} \equiv \mathrm{N}) ; 1,711(\mathrm{~s}, \mathrm{C}=\mathrm{O}) ; 1,534\left(\mathrm{~s}, \mathrm{NO}_{2}\right) ; 1,394\left(\mathrm{~s}, \mathrm{NO}_{2}\right) ; 1,350\left(\mathrm{~s}, \mathrm{~N}^{+} \mathrm{O}^{-}\right)$; 1,106 (m, Ar-F); Anal. Calc. for $\mathrm{C}_{14} \mathrm{H}_{5} \mathrm{~F}_{2} \mathrm{~N}_{5} \mathrm{O}_{6}$ : C: 44.58\%; H: 1.34\%; N: 18.57\%. Found: C: 44.04\%; $\mathrm{H}: 1.34 \%$; N $18.33 \%$.

5-Nitrofuran-2-carboxylic acid (7-trifluoromethyl-3-cyano-1,4-di-N-oxide-quinoxalin-2-yl)amide (33). Yield $39.8 \%$; ${ }^{1} \mathrm{H}-\mathrm{NMR} \delta$ ppm: $8.67\left(\mathrm{~s}, 1 \mathrm{H}, \mathrm{H}_{8}\right) ; 8.54\left(\mathrm{~d}, 1 \mathrm{H}, \mathrm{H}_{5}, J_{5-6}=8.9 \mathrm{~Hz}\right) ; 8.37$ (dd, $1 \mathrm{H}, \mathrm{H}_{6}, J_{6-8}=$ $1.6 \mathrm{~Hz}) ; 7.84\left(\mathrm{~s}, 2 \mathrm{H}, \mathrm{H}_{4^{\prime}}+\mathrm{H}_{3^{\prime}}\right)$; IR $\vee \mathrm{cm}^{-1}: 3,275(\mathrm{~m}, \mathrm{NH}) ; 2,244(\mathrm{w}, \mathrm{C} \equiv \mathrm{N}) ; 1,707(\mathrm{~s}, \mathrm{C}=\mathrm{O}) ; 1,536$ (s, $\left.\mathrm{NO}_{2}\right) ; 1,391\left(\mathrm{~s}, \mathrm{NO}_{2}\right) ; 1,350\left(\mathrm{~s}, \mathrm{~N}^{+} \mathrm{O}^{-}\right) ; 1,173\left(\mathrm{~m}, \mathrm{Ar}_{-} \mathrm{CF}_{3}\right)$; Anal. Calc. for $\mathrm{C}_{15} \mathrm{H}_{6} \mathrm{~F}_{3} \mathrm{~N}_{5} \mathrm{O}_{6}: \mathrm{C}: 44.02 \%$; H: $1.48 \%$; N: $17.11 \%$. Found: C: 44.08\%; H: 1.47\%; N: $16.71 \%$.

5-Nitrofuran-2-carboxylic acid (6-trifluoromethyl-3-cyano-1,4-di- $\mathrm{N}$-oxide-quinoxalin-2-yl)amide (34). Yield $51.8 \%$; ${ }^{1} \mathrm{H}-\mathrm{NMR} \delta$ ppm: $8.68\left(\mathrm{~s}, 1 \mathrm{H}, \mathrm{H}_{5}\right) ; 8.66\left(\mathrm{~d}, 1 \mathrm{H}, \mathrm{H}_{8}, J_{8-7}=9.1 \mathrm{~Hz}\right) ; 8.30\left(\mathrm{~d}, 1 \mathrm{H}, \mathrm{H}_{7}\right) ; 7.89$ $\left(\mathrm{d}, 1 \mathrm{H}, \mathrm{H}_{4^{\prime}}, J_{4^{\prime}-3^{\prime}}=3.9 \mathrm{~Hz}\right) ; 7.84\left(\mathrm{~d}, 1 \mathrm{H}, \mathrm{H}_{3^{\prime}}\right) . \mathrm{IR} \mathrm{cm}^{-1}: 3277(\mathrm{~m}, \mathrm{NH}) ; 2238(\mathrm{w}, \mathrm{C} \equiv \mathrm{N}) ; 1709(\mathrm{~s}, \mathrm{C}=\mathrm{O})$; $1538\left(\mathrm{~s}, \mathrm{NO}_{2}\right) ; 1391\left(\mathrm{~s}, \mathrm{NO}_{2}\right) ; 1350\left(\mathrm{~s}, \mathrm{~N}^{+} \mathrm{O}^{-}\right) ; 1173\left(\mathrm{~m}, \mathrm{Ar}-\mathrm{CF}_{3}\right)$. Calculated analysis for $\mathrm{C}_{15} \mathrm{H}_{6} \mathrm{~F}_{3} \mathrm{~N}_{5} \mathrm{O}_{6}$ : C:44.02\%; H:1.48\%; N:17.11\%. Found: C:44.26\%; H:1.61\%; N:16.43\%.

5-Nitrothiophene-2-carboxylic acid (3-cyano-1,4-di-N-oxide-quinoxalin-2-yl)amide (35). Yield 36.2 $\%$; ${ }^{1} \mathrm{H}-\mathrm{NMR} \delta$ ppm: $8.49\left(\mathrm{~s}, 1 \mathrm{H}, \mathrm{H}_{8}\right), 8.47\left(\mathrm{~s}, 1 \mathrm{H}, \mathrm{H}_{5}\right), 8.26-8.23\left(\mathrm{~m}, 2 \mathrm{H}, \mathrm{H}_{4},+\mathrm{H}_{3^{\prime}}\right) 8.11\left(\mathrm{dd}, 1 \mathrm{H}, \mathrm{H}_{6}, J_{6-}\right.$ $\left.{ }_{5}=8.3 \mathrm{~Hz}, J_{6-7}=7.4 \mathrm{~Hz}\right), 8.00\left(\mathrm{dd}, 1 \mathrm{H}, \mathrm{H}_{7}, J_{7-8}=8.1 \mathrm{~Hz}\right) ; \mathrm{IR} v \mathrm{~cm}^{-1}: 3,278(\mathrm{w}, \mathrm{NH}) ; 2,232(\mathrm{w}, \mathrm{C} \equiv \mathrm{N})$; $1,673(\mathrm{~s}, \mathrm{C}=\mathrm{O}) ; 1,538\left(\mathrm{~s}, \mathrm{NO}_{2}\right) ; 1,356\left(\mathrm{~m}, \mathrm{NO}_{2}\right) ; 1,333\left(\mathrm{~s}, \mathrm{~N}^{+} \mathrm{O}^{-}\right)$; Anal. Calc. for $\mathrm{C}_{14} \mathrm{H}_{7} \mathrm{~N}_{5} \mathrm{O}_{5} \mathrm{~S}: \mathrm{C}$ : 47.06\%; H: 1.97\%; N: 19.60\%. Found: C: 47,16\%; H: 2.31\%; N: 19.31\%.

5-Nitrothiophene-carboxylic acid (3-cyano-6-methyl-1,4-di- $N$-oxide-quinoxalin-2-yl)amide (36). Yield $41.3 \%{ }^{1} \mathrm{H}-\mathrm{NMR} \delta$ ppm: $8.41\left(\mathrm{~d}, 1 \mathrm{H}, \mathrm{H}_{8}, J_{8-7}=8.8 \mathrm{~Hz}\right) ; 8.31\left(\mathrm{~s}, 1 \mathrm{H}, \mathrm{H}_{5}\right) ; 8.25\left(\mathrm{~s}, 2 \mathrm{H}, \mathrm{H}_{3},+\mathrm{H}_{4}\right) ; 7.96$ $\left(\mathrm{dd}, 1 \mathrm{H}, \mathrm{H}_{7}, J_{7-8}=8.8 \mathrm{~Hz}, J_{7-5}=1.7 \mathrm{~Hz}\right) ; 2.61\left(\mathrm{~s}, 3 \mathrm{H}, \mathrm{CH}_{3}\right) ; \mathrm{IR} v \mathrm{~cm}^{-1}: 3,231(\mathrm{w}, \mathrm{NH}) ; 2,238(\mathrm{w}, \mathrm{C} \equiv \mathrm{N})$; $1,677(\mathrm{~s}, \mathrm{C}=\mathrm{O}) ; 1,528\left(\mathrm{~m}, \mathrm{NO}_{2}\right) ; 1,348\left(\mathrm{~m}, \mathrm{NO}_{2}\right) ; 1,325\left(\mathrm{~s}, \mathrm{~N}^{+} \mathrm{O}^{-}\right)$; Anal. Calc. for $\mathrm{C}_{15} \mathrm{H}_{9} \mathrm{~N}_{5} \mathrm{O}_{5} \mathrm{~S}: \mathrm{C}$ : 48.52\%; H: 2.44\%; N: 18.86\%. Found: C: 48.26\%; H: 2.48\%; N: 19.05\%. 
5-Nitrothiophene-2-carboxylic acid (3-cyano-6,7-dimethyl-1,4-di-N-oxide-quinoxalin-2-yl)amide (37). Yield $8.6 \%$; ${ }^{1} \mathrm{H}-\mathrm{NMR} \delta$ ppm: $8.29\left(\mathrm{~s}, 1 \mathrm{H}, \mathrm{H}_{8}\right) ; 8.28\left(\mathrm{~s}, 1 \mathrm{H}, \mathrm{H}_{5}\right) ; 8.24\left(\mathrm{~s}, 2 \mathrm{H}, \mathrm{H}_{3}+\mathrm{H}_{4}\right) ; 5.76(\mathrm{~s}, 1 \mathrm{H}$, $\mathrm{NH}) ; 2.54\left(\mathrm{~s}, 3 \mathrm{H}, \mathrm{CH}_{3}\right) ; 2.52\left(\mathrm{~s}, 3 \mathrm{H}, \mathrm{CH}_{3}\right) . \mathrm{IR} \vee \mathrm{cm}^{-1}: 3,244(\mathrm{w}, \mathrm{NH}) ; 2,238(\mathrm{w}, \mathrm{C} \equiv \mathrm{N}) ; 1,676(\mathrm{~m}$, $\mathrm{C}=\mathrm{O}) ; 1,528\left(\mathrm{~s}, \mathrm{NO}_{2}\right) ; 1,372\left(\mathrm{~m}, \mathrm{NO}_{2}\right) ; 1,332\left(\mathrm{~s}, \mathrm{~N}^{+} \mathrm{O}^{-}\right)$. Anal. Calc. for $\mathrm{C}_{16} \mathrm{H}_{11} \mathrm{~N}_{5} \mathrm{O}_{5} \mathrm{~S}: \mathrm{C}: 49.87 \% ; \mathrm{H}$ : 2.88\%; N:18.17\%. Found: C: 49.82\%; H: 2.98\%; N: 18.00\%.

5-Nitrothiophene-2-carboxylic acid (3-cyano-6-methoxy-1,4-di-N-oxide-quinoxalin-2-yl)amide (38). Yield $5.6 \%$; ${ }^{1} \mathrm{H}-\mathrm{NMR} \delta$ ppm: $8.43\left(\mathrm{~d}, 1 \mathrm{H}, \mathrm{H}_{8}, J_{8-7}=8.8 \mathrm{~Hz}\right) ; 8.22\left(\mathrm{~d}, 1 \mathrm{H}, \mathrm{H}_{4^{\prime}}, J_{4^{\prime}-3^{\prime}}=4.0 \mathrm{~Hz}\right) ; 8.16(\mathrm{~d}$, $\left.1 \mathrm{H}, \mathrm{H}_{3^{\prime}}\right) ; 7.77\left(\mathrm{~s}, 1 \mathrm{H}, \mathrm{H}_{5}\right) ; 7.72\left(\mathrm{~d}, 1 \mathrm{H}, \mathrm{H}_{7}\right) ; 4.03\left(\mathrm{~s}, 3 \mathrm{H}, \mathrm{OCH}_{3}\right) ; \mathrm{IR} v \mathrm{~cm}^{-1}: 3,270(\mathrm{w}, \mathrm{NH}) ; 2,238$ (w, $\mathrm{C} \equiv \mathrm{N}) ; 1,681(\mathrm{w}, \mathrm{C}=\mathrm{O}) ; 1,515\left(\mathrm{~s}, \mathrm{NO}_{2}\right) ; 1,348\left(\mathrm{~m}, \mathrm{NO}_{2}\right) ; 1,333\left(\mathrm{~s}, \mathrm{~N}^{+} \mathrm{O}^{-}\right)$; Anal. Calc. for $\mathrm{C}_{15} \mathrm{H}_{9} \mathrm{~N}_{5} \mathrm{O}_{6} \mathrm{~S}$ : C: $46.51 \%$; H: $2.34 \%$; N: 18.08\%. Found: C: 46.64\%; H: 2.52\%; N: 18.28\%.

5-Nitrothiophene-2-carboxylic acid (6-chloro-3-cyano-1,4-di-N-oxide-quinoxalin-2-yl)amide (39). Yield $36.7 \%$; ${ }^{1} \mathrm{H}-\mathrm{NMR} \delta$ ppm: $8.50\left(\mathrm{~d}, 1 \mathrm{H}, \mathrm{H}_{5}, J_{5-7}=2.2 \mathrm{~Hz}\right) ; 8.48\left(\mathrm{~d}, 1 \mathrm{H}, \mathrm{H}_{8}, J_{8-7}=9.2 \mathrm{~Hz}\right) ; 8.24(\mathrm{~d}$, $\left.1 \mathrm{H}, \mathrm{H}_{4^{\prime}}, J_{4^{\prime}-3^{\prime}}=4.4 \mathrm{~Hz}\right) ; 8.23\left(\mathrm{~d}, 1 \mathrm{H}, \mathrm{H}_{3^{\prime}}\right) ; 8.14\left(\mathrm{dd}, 1 \mathrm{H}, \mathrm{H}_{7}, J_{7-8}=9.2 \mathrm{~Hz}, J_{7-5}=2.2 \mathrm{~Hz}\right) ; \mathrm{IR} v \mathrm{~cm}^{-1}$ : 3,275 (w, NH); $2,239(\mathrm{w}, \mathrm{C} \equiv \mathrm{N}) ; 1,677(\mathrm{~s}, \mathrm{C}=\mathrm{O}) ; 1,519\left(\mathrm{~s}, \mathrm{NO}_{2}\right) ; 1,357\left(\mathrm{~m}, \mathrm{NO}_{2}\right) ; 1,323\left(\mathrm{~s}, \mathrm{~N}^{+} \mathrm{O}^{-}\right)$; 1,090 (m, Ar-Cl); Anal. Calc. for $\mathrm{C}_{14} \mathrm{H}_{6} \mathrm{ClN}_{5} \mathrm{O}_{5} \mathrm{~S}: \mathrm{C}: 42.92 \%$; $\mathrm{H}: 1.54 \%$; N: 17.88\%. Found: C: $43.01 \%$; H: $1.71 \%$; N: $18.02 \%$.

5-Nitrothiophene-2-carboxylic acid (6,7-dichloro-3-cyano-1,4-di-N-oxide-quinoxalin-2-yl)amide (40). Yield $4.4 \%$; ${ }^{1} \mathrm{H}-\mathrm{NMR} \delta$ ppm: $8.66\left(\mathrm{~s}, 1 \mathrm{H}, \mathrm{H}_{8}\right) ; 8.60\left(\mathrm{~s}, 1 \mathrm{H}, \mathrm{H}_{5}\right) ; 8.19\left(\mathrm{~s}, 1 \mathrm{H}, \mathrm{H}_{4}\right) ; 8.05\left(\mathrm{~s}, 1 \mathrm{H}, \mathrm{H}_{3}{ }\right)$; IR $v \mathrm{~cm}^{-1}: 3,256(\mathrm{w}, \mathrm{NH}) ; 2,238(\mathrm{w}, \mathrm{C} \equiv \mathrm{N}) ; 1,675(\mathrm{~m}, \mathrm{C}=\mathrm{O}) ; 1,505\left(\mathrm{~s}, \mathrm{NO}_{2}\right) ; 1,354\left(\mathrm{~m}, \mathrm{NO}_{2}\right) ; 1,330$ (s, $\mathrm{N}^{+} \mathrm{O}^{-}$); Anal. Calc. for $\mathrm{C}_{14} \mathrm{H}_{5} \mathrm{Cl}_{2} \mathrm{~N}_{5} \mathrm{O}_{5} \mathrm{~S}: \mathrm{C}: 39.46 \%$; $\mathrm{H}: 1.18 \%$; $: 16.43 \%$. Found: C: 39.00\%; H: $1.35 \% ; \mathrm{N}: 16.07 \%$.

5-Nitrothiophene-2-carboxylic acid (6-fluoro-3-cyano-1,4-di-N-oxide-quinoxalin-2-yl)amide (41). Yield 24.0 \%; ${ }^{1} \mathrm{H}-\mathrm{NMR} \delta$ ppm: 8.60-8.56 (m, $\left.1 \mathrm{H}, \mathrm{H}_{8}\right) ; 8.32-8.29\left(\mathrm{~m}, 1 \mathrm{H}, \mathrm{H}_{5}\right) ; 8.25\left(\mathrm{~s}, 2 \mathrm{H}, \mathrm{H}_{4^{\prime}}+\mathrm{H}_{3^{\prime}}\right)$; 8.07-8.02 (m, 1H, $\left.\mathrm{H}_{6}\right) ; 5.76(\mathrm{~s}, 1 \mathrm{H}, \mathrm{NH})$; IR $v \mathrm{~cm}^{-1}: 3,293(\mathrm{~m}, \mathrm{NH}) ; 2,238(\mathrm{w}, \mathrm{C} \equiv \mathrm{N}) ; 1,671(\mathrm{~s}, \mathrm{C}=\mathrm{O})$; $1,514\left(\mathrm{~s}, \mathrm{NO}_{2}\right) ; 1,349\left(\mathrm{~m}, \mathrm{NO}_{2}\right) ; 1,332\left(\mathrm{~s}, \mathrm{~N}^{+} \mathrm{O}^{-}\right) ; 1,114(\mathrm{~m}, \mathrm{Ar}-\mathrm{F})$; Anal. Calc. for $\mathrm{C}_{14} \mathrm{H}_{6} \mathrm{FN}_{5} \mathrm{O}_{5} \mathrm{~S}: \mathrm{C}$ : 44.81\%; H: 1.61\%; N: 18.66\%. Found: C: 44.31\%; H: 1.69\%; N: 18.44\%.

5-Nitrothiophene-2-carboxylic acid (6,7-difluoro-3-cyano-1,4-di-N-oxide-quinoxalin-2-yl)amide (42). Yield $33.4 \%$; ${ }^{1} \mathrm{H}-\mathrm{NMR} \delta$ ppm: 8.65-8.55 (m, 2H, $\left.\mathrm{H}_{8}+\mathrm{H}_{5}\right) ; 8.24\left(\mathrm{~d}, 1 \mathrm{H}, \mathrm{H}_{4^{\prime}}, J_{4^{\prime}-3^{\prime}}=4.5 \mathrm{~Hz}\right) ; 8.23(\mathrm{~d}$, $\left.1 \mathrm{H}, \mathrm{H}_{3^{\prime}}\right)$; IR $v \mathrm{~cm}^{-1}: 3,256(\mathrm{w}, \mathrm{NH}) ; 2,232(\mathrm{w}, \mathrm{C} \equiv \mathrm{N}) ; 1,685(\mathrm{~s}, \mathrm{C}=\mathrm{O}) ; 1,517\left(\mathrm{~s}, \mathrm{NO}_{2}\right) ; 1,359\left(\mathrm{~s}, \mathrm{NO}_{2}\right)$; $1,341\left(\mathrm{~s}, \mathrm{~N}^{+} \mathrm{O}^{-}\right)$; Anal. Calc. for $\mathrm{C}_{14} \mathrm{H}_{5} \mathrm{~F}_{2} \mathrm{~N}_{5} \mathrm{O}_{5} \mathrm{~S}: \mathrm{C}: 42.76 \%$; H: 1.28\%; N: 17.81\%. Found: C: 42.78\%; $\mathrm{H}: 1.30 \%$; N $17.89 \%$.

5-Nitrothiophene-2-carboxylic acid (7-trifluoromethyl-3-cyano-1,4-di- $\mathrm{N}$-oxide-quinoxalin-2-yl)amide (43). Yield $51.8 \%$; ${ }^{1} \mathrm{H}-\mathrm{NMR} \delta$ ppm: $8.65\left(\mathrm{~d}, 1 \mathrm{H}, \mathrm{H}_{5}, J_{5-6}=8.8 \mathrm{~Hz}\right) ; 8.65\left(\mathrm{~s}, 1 \mathrm{H}, \mathrm{H}_{8}\right) ; 8.27$ (dd, $1 \mathrm{H}, \mathrm{H}_{6}$, $\left.J_{6-8}=1.5 \mathrm{~Hz}\right) ; 8.23\left(\mathrm{~d}, 1 \mathrm{H}, \mathrm{H}_{4^{\prime}}, J_{4^{\prime}-3^{\prime}}=4.4 \mathrm{~Hz}\right) ; 8.18\left(\mathrm{~d}, 1 \mathrm{H}, \mathrm{H}_{3^{\prime}}\right) ; \mathrm{IR} v \mathrm{~cm}^{-1}: 3,244(\mathrm{w}, \mathrm{NH}) ; 2,238$ (w, $\mathrm{C} \equiv \mathrm{N}) ; 1,672(\mathrm{~s}, \mathrm{C}=\mathrm{O}) ; 1,506\left(\mathrm{~s}, \mathrm{NO}_{2}\right) ; 1,399\left(\mathrm{~s}, \mathrm{NO}_{2}\right) ; 1,338\left(\mathrm{~s}, \mathrm{~N}^{+} \mathrm{O}^{-}\right) ; 1,130\left(\mathrm{~m}, \mathrm{Ar}-\mathrm{CF}_{3}\right)$; Anal. Calc. for $\mathrm{C}_{15} \mathrm{H}_{6} \mathrm{~F}_{3} \mathrm{~N}_{5} \mathrm{O}_{5} \mathrm{~S}$ : C: $42.36 \%$; H: $1.42 \%$; N: 16.47\%. Found: C: $42.58 \%$; H: $1.60 \%$; N: $16.49 \%$. 
5-Nitrothiophene-2-carboxylic acid (6-trifluoromethyl-3-cyano-1,4-di- $\mathrm{N}$-oxide-quinoxalin-2-yl)amide (44). Yield $57.9 \%$; ${ }^{1} \mathrm{H}-\mathrm{NMR} \delta$ ppm: 8.64 (s, $\left.1 \mathrm{H}, \mathrm{H}_{5}\right) ; 8.47$ (d, $\left.1 \mathrm{H}, \mathrm{H}_{8}, J_{8-7}=8.9 \mathrm{~Hz}\right) ; 8.35$ (d, $\left.1 \mathrm{H}, \mathrm{H}_{7}\right)$; $8.20\left(\mathrm{~d}, 1 \mathrm{H}, \mathrm{H}_{4^{\prime}}, J_{4^{\prime}-3^{\prime}}=4.3 \mathrm{~Hz}\right) ; 8.08\left(\mathrm{~d}, 1 \mathrm{H}, \mathrm{H}_{3}{ }^{\prime}\right) ; \mathrm{IR} \vee \mathrm{cm}^{-1}: 3,247(\mathrm{w}, \mathrm{NH}) ; 2,238(\mathrm{w}, \mathrm{C} \equiv \mathrm{N}) ; 1,675(\mathrm{~s}$, $\mathrm{C}=\mathrm{O}) ; 1,520\left(\mathrm{~s}, \mathrm{NO}_{2}\right) ; 1,399\left(\mathrm{~m}, \mathrm{NO}_{2}\right) ; 1,333\left(\mathrm{~s}, \mathrm{~N}^{+} \mathrm{O}^{-}\right) ; 1,139\left(\mathrm{~m}, \mathrm{Ar}-\mathrm{CF}_{3}\right)$; Anal. Calc. for $\mathrm{C}_{15} \mathrm{H}_{6} \mathrm{~F}_{3} \mathrm{~N}_{5} \mathrm{O}_{5} \mathrm{~S}$ : C: 42.36\%; H: 1.42\%; N: 16.47\%. Found: C: 42.62\%; H: 1.72\%; N: 16.57\%.

In vitro evaluation of antituberculosis activity

In vitro evaluation of the antituberculosis activity was carried out at the GWL Hansen's Disease Center within the Tuberculosis Antimicrobial Acquisition \& Coordinating Facility (TAACF) screening program for the discovery of novel drugs for the treatment of tuberculosis. The Southern Research Institute coordinates the overall program under the direction of the U.S. National Institute of Allergy and Infectious Disease (NIAID). The purpose of the screening program is to provide a resource whereby new experimental compounds can be tested for their capability to inhibit the growth of virulent M. tuberculosis [26].

Determination of growth inhibition percentage via MABA: The initial screen is conducted against Mycobacterium tuberculosis H37Rv (ATCC 27294) in BACTEC 12B medium using the Microplate Alamar Blue Assay (MABA) [27]. The fluorescence changes due to the reduction of Alamar blue dye during the growth of Mycobacterium were monitored by the BACTEC 460-radiometric system. Compounds effecting $<90 \%$ inhibition in the primary screen (MIC $>6.25 \mu \mathrm{g} / \mathrm{mL}$ ) were not further evaluated.

Determination of minimum inhibitory concentration (MIC) via MABA: Compounds demonstrating at least $90 \%$ inhibition in the primary screen were re-tested against $M$. tuberculosis $\mathrm{H} 37 \mathrm{Rv}$ at lower concentrations in order to determine the actual minimum inhibitory concentration (MIC) in the MABA. The MIC was defined as the lowest concentration effecting a reduction in fluorescence of $90 \%$ relative to controls. RIF was used as the reference compound (RIF MIC $=0.015-0.125 \mu \mathrm{g} / \mathrm{mL}$ ).

In vitro evaluation of trypanocidal activity

Trypanosoma cruzi epimastigotes (Tulahuen 2 strain) were grown at $28{ }^{\circ} \mathrm{C}$ in an axenic medium (BHI-Tryptose) as previously described [28-30], supplemented with 5\% fetal bovine serum (FBS). Cells from a 10-day-old culture (stationary phase) were inoculated into $50 \mathrm{~mL}$ of fresh culture medium in order to give an initial concentration of $1 \times 10^{6}$ cells $/ \mathrm{mL}$. Cell growth was followed by measuring the absorbance of the culture at $600 \mathrm{~nm}$ daily. Before inoculation, the medium was supplemented with the indicated amount of the drug from a stock solution in DMSO. The final concentration of DMSO in the culture medium never exceeded $0.4 \%$, and the control was run in the presence of $0.4 \%$ DMSO and in the absence of any drug. No effect on epimastigote growth was observed by the presence of up to $1 \%$ DMSO in the culture medium. The percentage of growth inhibition (PGI) was calculated as follows: PGI $(\%)=\{1-[(\mathrm{Ap}-\mathrm{A} 0 \mathrm{p}) /(\mathrm{Ac}-\mathrm{A} 0 \mathrm{c})]\} \times 100$, where $\mathrm{Ap}=\mathrm{A}_{600}$ of the culture containing the drug at day 5; $\mathrm{A} 0 \mathrm{p}=\mathrm{A}_{600}$ of the culture containing the drug just after addition of the inocula (day 0 ); $\mathrm{Ac}=\mathrm{A}_{600}$ of the culture in the absence of the drug (control) at day 5; $\mathrm{A} 0 \mathrm{c}=\mathrm{A}_{600}$ in the absence of 
the drug at day 0. In order to determine $\mathrm{IC}_{50}$ values, $50 \%$ inhibitory concentrations, parasite growth was observed in the absence (control) and presence of increasing concentrations of the corresponding drug. At day 5, the absorbance of the culture was measured and related to the control. The $\mathrm{IC}_{50}$ value was considered to be the concentration of drug needed for reducing the absorbance ratio to $50 \%$.

\section{Conclusions}

New structural modifications on the QDO skeleton were performed, and promising biological results against $M$. tuberculosis and T. cruzi were obtained. The biological evaluation showed a broad range of activities, thereby showing new structural hits for future chemical pharmacomodulations of QDO for the development of new drugs against tuberculosis and Chagas disease.

\section{Acknowledgements}

This work has been carried out with the financial support of the RIDIMEDCHAG-CYTED. We also wish to express our gratitude to the Tuberculosis Antimicrobial Acquisition \& Coordinating Facility (TAACF) for the evaluation of the antituberculosis activity through research and development contracts. S.A. is indebted to the Navarra Government for a grant. E. M. is indebted to the La Rioja Government for a grant. D.B. is a PEDECIBA student.

\section{References and Notes}

1. TAACF: http://www.taacf.org/about-TB-background.htm. Accessed May 5, 2009.

2. NIAID: http://www3.niaid.nih.gov/topics/tuberculosis/. Accessed May 5, 2009.

3. Jaso, A.; Zarranz, B.; Aldana, I.; Monge, A. Synthesis of new quinoxaline-2-carboxylate 1,4dioxide derivatives as anti-Mycobacterium tuberculosis agents. J. Med. Chem. 2005, 48, 20192025.

4. Tangallapally, R.P.; Yendapally, R.; Lee, R.E.; Hevener, K.; Jones, V.C.; Lenaerts, A.J.; McNeil, M.R.; Wang, Y.; Franzblau, S.; Lee, R.E. Synthesis and evaluation of nitrofuranylamides as novel antituberculosis agents. J. Med. Chem. 2004, 47, 5276-5283.

5. WHO: http://www.who.int/neglected_diseases/diseases/chagas/en/index.html. Accessed June 14, 2009.

6. Cerecetto, H.; González, M. Chemotherapy of Chagas' disease: Status and new developments. Curr. Top. Med. Chem. 2002. 2, 1187-1213.

7. González, M.; Cerecetto, H.; Monge, A. Quinoxaline 1,4-dioxide and Phenazine 5,10-dioxide. Chemistry and Biology. In Topics in Heterocyclic Chemistry. Bioactive Heterocycles V; Khan, M.T.H., Ed.; Springer: Berlin, Germany, 2007; Volume 11, p. 179-211.

8. Ortega, M.A.; Sainz, Y.; Montoya, M.E.; López de Ceráin, A.; Monge, A. Synthesis and antituberculosis activity of new 2-quinoxalinecarbonitrile 1,4-di- $N$-oxides. Pharmazie 1999, 54, 24-25.

9. Sainz, Y.; Montoya, M.E.; Martínez-Crespo, F.J.; Ortega, M.A.; López de Ceráin, A.; Monge, A. New quinoxaline 1,4-di- $N$-oxides for treatment of tuberculosis. Arzneimittelforschung 1999, 49, 55-59. 
10. Ortega, M.A.; Montoya, M.E.; Jaso, A.; Zarranz, B.; Tirapu, I.; Aldana, I.; Monge, A. Antimycobacterial activity of new quinoxaline-2-carbonitrile and quinoxaline-2-carbonitrile 1,4di-N-oxide derivatives. Pharmazie 2001, 56, 205-207.

11. Ortega, M.A.; Sainz, Y.; Montoya, M.E.; Jaso, A.; Zarranz, B.; Aldana, I.; Monge, A. AntiMycobacterium tuberculosis agents derived from quinoxaline-2-carbonitrile and quinoxaline-2carbonitrile 1,4-di-N-oxide. Arzneimittelforschung 2002, 52, 113-119.

12. Zarranz, B.; Jaso, A.; Aldana, I.; Monge, A. Synthesis and antimycobacterial activity of new quinoxaline-2-carboxamide 1,4-di- $N$-oxide derivatives. Bioorg. Med. Chem. 2003, 11, 2149-2156.

13. Jaso, A.; Zarranz, B.; Aldana, I.; Monge, A. Synthesis of new 2-acetyl and 2-benzoyl quinoxaline 1,4-di- $N$-oxide derivatives as anti-Mycobacterium tuberculosis agents. Eur. J. Med. Chem. 2003, 38, 791-800.

14. Villar, R.; Vicente, E.; Solano, B.; Pérez-Silanes, S.; Aldana, I.; Maddry, J.A.; Lenaerts, A.J.; Franzblau, S.G.; Cho, S.H.; Monge, A.; Goldman, R.C. In vitro and in vivo antimycobacterial activities of ketone and amide derivatives of quinoxaline 1,4-di- $N$-oxide. J. Antimicrob. Chemother. 2008, 62, 547-554.

15. Vicente, E.; Villar, R.; Burguete, A.; Solano, B.; Pérez-Silanes, S.; Aldana, I.; Maddry, J.A.; Lenaerts, A.J.; Franzblau, S.G.; Cho, S.H.; Monge, A.; Goldman, R.C. Efficacy of quinoxaline-2carboxylate 1,4-di- $N$-oxide derivatives in experimental tuberculosis. Antimicrob. Agents Chemother. 2008, 52, 3321-3326.

16. Vicente, E.; Pérez-Silanes, S.; Lima, L.M.; Ancizu, S.; Burguete, A.; Solano, B.; Villar, R.; Aldana, I.; Monge, A. Selective activity against Mycobacterium tuberculosis of new quinoxaline 1,4-di-N-oxides. Bioorg. Med. Chem. 2009, 17, 385-389.

17. Aguirre, G.; Cerecetto, H.; Di Maio, R.; González, M.; Montoya Alfaro, M.E.; Jaso, A.; Zarranz, B.; Ortega, M.A.; Aldana, I.; Monge-Vega, A. Quinoxaline $N, N^{\prime}$-dioxide derivatives and related compounds as growth inhibitors of Trypanosoma cruzi. Structure-activity relationships. Bioorg. Med. Chem. Lett. 2004, 14, 3835-3839.

18. Lavaggi, M.L.; Aguirre, G.; Boiani, L.; Orelli, L.; García, B.; Cerecetto, H.; González, M. Pyrimido[1,2-a]quinoxaline 6-oxide and phenazine 5,10-dioxide derivatives and related compounds as growth inhibitors of Trypanosoma cruzi. Eur. J. Med. Chem. 2008 43, 1737-1741.

19. Vicente, E.; Duchowicz, P.R.; Castro, E.A.; Monge, A. QSAR analysis for quinoxaline-2carboxylate 1,4-di- $N$-oxides as anti-mycobacterial agents. J. Mol. Graph. Model. 2009, doi:10.1016/j.jmgm.2009.03.004.

20. Vicente, E.; Villar, R.; Burguete, A.; Solano, B.; Ancizu, S.; Pérez-Silanes, S.; Aldana, I.; Monge, A. Substitutions of fluorine atoms and phenoxy groups in the synthesis of quinoxaline 1,4-di- $N$ oxide derivatives. Molecules 2008, 13, 86-95.

21. Cerecetto, H.; González, M. Anti-T. cruzi agents: our experience in the evaluation of more than five hundred compounds. Mini Rev. Med. Chem. 2008, 8, 1355-1383.

22. González, M.; Cerecetto, H. Benzofuroxan and Furoxan. Chemistry and Biology. In Topics in Heterocyclic Chemistry. Bioactive Heterocycles V.; Khan, M.T.H, Ed.; Springer: Berlin, Germany, 2007; Volume 10, pp. 265-308.

23. Ley, K.; Seng. F. Synthesis unter verwendung von benzofuroxan. Synthesis 1975, 415-422. 
24. Dellaria, J.F.; Sallin K.J.; Rodriques, K. Synthesis of new amide-linked N-hydroxyurea 5lipoxygenase inhibitors by an intramolecular oxygen to nitrogen acyl transfer. Bioorg. Med. Chem. Lett. 1993, 3, 305-308.

25. Cheeseman G.W.H.; Cookson, R.F. In Condensed Pyrazines. Wiley and Sons: New York, NY, USA, 1979; p. 35.

26. TAACF: http://www.taacf.org/Process-text.htm\#assays. Accessed 5 May, 2009.

27. Collins, L.A.; Franzblau, S.G. Microplate Alamar Blue Assay versus BACTEC 460 System for High-Throughput Screening of Compounds against Mycobacterium tuberculosis and Mycobacterium avium. Antimicrob. Antimicrob. Agents Chemother. 1997, 41, 1004-1009.

28. Porcal, W.; Hernández, P.; Boiani, M.; Aguirre, G.; Boiani, L.; Chidichimo, A.; Cazzulo, J.J.; Campillo, N.E.; Paez, J.A.; Castro, A.; Krauth-Siegel, R.L.; Davies, C.; Basombrío, M.A.; González, M.; Cerecetto, H. In vivo anti-Chagas vinylthio-, vinylsulfinyl-, and vinylsulfonylbenzofuroxan derivatives. J. Med. Chem. 2007, 50, 6004-6015.

29. Rodríguez, J.; Gerpe, A.; Aguirre, G.; Kemmerling, U.; Piro, O.E.; Arán, V.J.; Maya, J.D.; OleaAzar, C.; González, M.; Cerecetto, H. Study of 5-nitroindazoles' anti-Trypanosoma cruzi mode of action: electrochemical behaviour and ESR spectroscopic studies. Eur. J. Med. Chem. 2009, 44, 1545-1553.

30. Bollini, M.; Casal, J.J.; Alvarez, D.E.; Boiani, L.; González, M.; Cerecetto, H.; Bruno, A.M. New potent imidazoisoquinolinone derivatives as anti-Trypanosoma cruzi agents: biological evaluation and structure-activity relationships. Bioorg. Med. Chem. 2009, 17, 1437-1444.

Sample Availability: Contact the authors.

(C) 2009 by the authors; licensee Molecular Diversity Preservation International, Basel, Switzerland. This article is an open-access article distributed under the terms and conditions of the Creative Commons Attribution license (http://creativecommons.org/licenses/by/3.0/). 